\title{
Multi-object Tracking for Generic Observation Model Using Labeled Random Finite Sets
}

\author{
Suqi Li, Wei Yi*, Reza Hoseinnezhad, Bailu Wang, Lingjiang Kong
}

\begin{abstract}
This paper presents an exact Bayesian filtering solution for the multi-object tracking problem with the generic observation model. The proposed solution is designed in the labeled random finite set framework, using the product styled representation of labeled multi-object densities, with the standard multi-object transition kernel and no particular simplifying assumptions on the multi-object likelihood. Computationally tractable solutions are also devised by applying a principled approximation involving the replacement of the full multi-object density with a labeled multi-Bernoulli density that minimizes the Kullback-Leibler divergence and preserves the first-order moment. To achieve the fast performance, a dynamic grouping procedure based implementation is presented with a step-bystep algorithm. The performance of the proposed filter and its tractable implementations are verified and compared with the state-of-the-art in numerical experiments.
\end{abstract}

\section{INTRODUCTION}

Finite set statistics (FISST) [1] has become a hot spot in multi-object inference for the random finite set (RFS) framework can perfectly accommodate relatively accurate models for the behavior of multi-object dynamic systems, especially in terms of its ability to capture the randomness of both the number of, and the values of object states, as well as their statistical correlations. FISST has attracted substantial interest from academia as well as the commercial sector with applications spanning many areas such as, biology [2], physics [3], computer vision [4], multi-object tracking [5][7], and robotics [8]. At the core of multi-object tracking is Bayes filter which is usually intractable due to suffering from the curse of dimensionality with computing set integrals and the combinatorial growth of computations involved with increasing number of objects. In order to solve these problems, several tractable approximations of multi-object Bayes filter have been proposed successively, namely the probability hypothesis density (PHD) filter [5], [9], the cardinalized PHD filter [10], [11], and the multi-Bernoulli filter [1], [12], [13].

This work was supported by the Chang Jiang Scholars Program, the National Natural Science Foundation of China under Grant 61771110, the Fundamental Research Funds of Central Universities under Grants ZYGX2016J031, the Chinese Postdoctoral Science Foundation under Grant 2014M550465 and Special Grant 2016T90845, and the Australian Research Council (ARC) through the Linkage Project Grant LP160101081. (Corresponding author: Wei Yi.)

S. Li, B. Wang, W. Yi, and L. Kong are with the School of Electronic Engineering, University of Electronic Science and Technology of China, Chengdu 611731, China (Email: qi_qi_zhu1210@163.com; kussoyi@gmail.com; w_b_13020@163.com; lingjiang.kong@gmail.com).

R. Hoseinnezhad is with the School of Aerospace, Mechanical and Manufacturing Engineering, RMIT University, Victoria 3083, Australia (Email: reza.hoseinnezhad@rmit.edu.au).
With the recent development of labeled set filters [14][22] and their enhanced performance compared to previous unlabeled versions, the study on the FISST-based multi-object tracking has recently turned its focus on the labeled random set filters. Vo et al. [14] proposed a class of generalized labeled multi-Bernoulli (GLMB) densities 1 and the relevant tracking filter, the GLMB filter. The advantages of GLMB RFS family are that they are conjugate priors with standard multi-object likelihood, and are closed under the multi-object ChapmanKolmogorov equation with respect to the standard multi-object transition kernel. Nevertheless, the $\delta$-GLMB filter involves exponential growth in the number of posterior components with the number of objects and therefore, tractable techniques for truncating the posterior and prediction densities are also proposed in [15]. Later, to further decrease the computational costs, principled approximations of the GLMB filter were proposed, including the labeled multi-Bernoulli (LMB) filter [18] and the marginalized $\delta$-GLMB filter [16]. These two filters are not only computationally cheaper, but also preserve the key statistical properties of the full multi-object posterior density.

All of the aforementioned labeled set filters are originally designed for the standard observation model, and are not necessarily suitable for the generic observation model (GOM) which involves no simplifying assumptions made on the multi-object likelihood. In many applications, there might be sensor observations that cannot be accurately modelled by the standard multi-object likelihood. Examples include the track-before-detect (TBD) problem [13], [23]-[28], superpositional sensors [20], [29]-[31], merged observations [17], and extended objects [32]. Sensors providing such non-standard observations are widely used in applications such as vehicle tracking using automotive radars, person tracking using laser sensors, acoustic amplitude sensors [33], and video tracking [4], [34]. Consequently, there is a substantial demand for devising multi-object tracking algorithms that work with the GOM.

There is no specified class of labeled RFSs that can be closed under the Bays' rule with respect to the GOM. In an independent work from this paper, Papi et al. [19] proposed a decomposition of the general case of the labeled multi-object (LMO) density, as the product of the joint existence probability of the label set and the joint probability density of states conditional on their corresponding labels. This decomposition provides an explicit expression for the LMO density, and is fundamental in the labeled multi-object filtering context

\footnotetext{
${ }^{1}$ GLMB distribution was also termed as Vo-Vo distribution by Mahler in his book [6].
} 
especially with the GOM. Papi et al. [19] also proposed an extension of the $\delta$-GLMB filter that works with the GOM, by replacing the multi-object posterior with a principled $\delta$-GLMB density approximation that minimizes the Kullback-Leibler divergence (KLD), and preserves the cardinality distribution and the first-order moment. To distinguish it from the conventional $\delta$-GLMB filter, it is referred to as the $\delta$-GLMB-GOM filter in this paper.

Unlike the $\delta$-GLMB-GOM filter which is an approximate solution for the multi-object tracking problem with the GOM, the novel solution presented in this paper, the LMO-GOM filter ${ }^{2}$ is an exact solution for the same problem. The prediction equations of the LMO-GOM filter are exact under the standard multi-object transition kernel which embeds the basic assumptions commonly made with multi-object tracking solutions, such as Markovian dynamics for object states, and the independence of the birth process from other object states. The update equations of the LMO-GOM filter are not based on any approximations or simplifying assumptions with the multi-object likelihood model. Essentially, the $\delta$-GLMB-GOM filter is an approximation of the LMO-GOM filter with the multi-object posterior approximated as a principled $\delta$-GLMB density.

Another major contribution is a generalization of the LMB filter, called the LMB-GOM filter, that works with generic multi-object likelihoods. The LMB-GOM filter is devised by approximating the original multi-object posterior with the closest LMB density in terms of its KLD. The approximate LMB density also matches the first-order moment of the original multi-object posterior. Our analysis shows that the computational cost of the LMB-GOM filter is less than the $\delta$-GLMB-GOM filter.

A third major contribution of is this paper is a variant of the proposed LMB filter, called the grouping based LMBGOM (G-LMB-GOM) filter which is essentially an efficient implementation of the LMB-GOM filter. The G-LMB-GOM filter is based on a dynamic grouping procedure which enables parallelization. This parallel implementation significantly reduces both the number and the dimension of integrals, leading to a substantial improvement in computational costs as well as the numerical accuracy when the computing and memory resources are limited. In some cases, the resulting improvements in the numerical accuracy are well beyond the extent of inaccuracies stemmed from the grouping procedure.

The performance of the proposed algorithms including the LMO-GOM and LMB-GOM/G-LMB-GOM filters, implemented via Sequential Monte Carlo (SMC) method, are presented and demonstrated in numerical experiments.

The rest of the paper is organized as follows. A background on notations, labeled RFSs and the formal statement of the labeled multi-object tracking problem is provided in Section II. Section III proposes the LMO-GOM filter and Section IV presents the "best" LMB approximation for the general LMO density and the resulting LMB-GOM filter. Section $\mathrm{V}$ provides a comparative summary for different labeled multi-object

\footnotetext{
${ }^{2}$ Preliminary results have been published in 35 . This paper provides a complete and detailed picture with extended results, proofs, and experiments.
}

tracking algorithms with the GOM. Section VI demonstrates the performance of the proposed algorithms via numerical experiments. Conclusions are remarked in Section VII

\section{BACKGROUND}

\section{A. Notations}

We adhere to the convention that single-object states are represented by lowercase letters, e.g., $\mathbf{x}, x$, while multi-object states are represented by uppercase letters, e.g., $\mathbf{X}, X$. To distinguish labeled states and distributions from the unlabeled ones, bold-type letters are adopted for the labeled ones, e.g., $\mathbf{X}, \mathbf{X}, \boldsymbol{\pi}$. Moreover, blackboard bold letters represent spaces, e.g., the state space is represented by $\mathbb{X}$, the label space by $\mathbb{L}$. The collection of all finite subsets of $\mathbb{X}$ is denoted by $\mathcal{F}(\mathbb{X})$.

The labeled single-object state $\mathbf{x}$ is constructed by augmenting a state $x \in \mathbb{X}$ with a label $\ell \in \mathbb{L}$. The labels are usually drawn from a discrete label space, $\mathbb{L}=\left\{\alpha_{i}, i \in \mathbb{N}\right\}$, where all $\alpha_{i} \mathrm{~s}$ are distinct and $\mathbb{N}$ is the set of positive integers.

We use the multi-object exponential notation

$$
h^{X} \triangleq \prod_{x \in X} h(x)
$$

for real-valued function $h$, with $h^{\emptyset}=1$ by convention.

To admit arbitrary arguments like sets, vectors and integers, the generalized Kronecker delta function and the inclusion function are repectively given by

$$
\delta_{Y}(X) \triangleq\left\{\begin{array} { l } 
{ 1 , \text { if } X = Y } \\
{ 0 , \text { otherwise, } }
\end{array} 1 _ { Y } ( X ) \triangleq \left\{\begin{array}{l}
1, \text { if } X \subseteq Y \\
0, \text { otherwise. }
\end{array}\right.\right.
$$

If $X$ is a singleton, i.e., $X=\{x\}$, the notation $1_{Y}(x)$ is used instead of $1_{Y}(\{x\})$. For functions $a(\mathbf{x})$ and $b(\mathbf{x})$ defined on $\mathbb{X} \times \mathbb{L}$, the inner product is denoted by $\langle a, b\rangle=\int a(\mathbf{x}) b(\mathbf{x}) d \mathbf{x}$.

\section{B. Labeled RFS}

The notion of labeled RFSs was firstly proposed in [14] to address the uniqueness of tracks. A labeled RFS [14], [15] with (kinematic) state space $\mathbb{X}$ and (discrete) label space $\mathbb{L}$ is an RFS on $\mathbb{X} \times \mathbb{L}$ such that each realization $\mathbf{X}$ has distinct labels. Let $\mathcal{L}: \mathbb{X} \times \mathbb{L} \rightarrow \mathbb{L}$ be the projection $\mathcal{L}((x, \ell))=\ell$, and hence $\mathcal{L}(\mathbf{X})=\{\mathcal{L}(\mathbf{x}), \mathbf{x} \in \mathbf{X}\}$ is the set of labels of $\mathbf{X}$. A labeled RFS and the set of its labels have the same cardinality, namely, $|\mathcal{L}(\mathbf{X})|=|\mathbf{X}|$. The function $\Delta(\mathbf{X})=\delta_{|\mathbf{X}|}(\mathcal{L}(\mathbf{X}))$ is called the distinct label indicator.

1) Decomposition of LMO Density: For an arbitrary labeled RFS, its multi-object density can be decomposed as the product of the joint existence probability of the label set and the joint probability density of states conditional on their corresponding labels [19]. The definitions of necessary quantities and the decomposition of the LMO density are briefly reviewed by providing a more rigorous definition.

The set of labels $\mathcal{L}(\mathbf{X})$ of a labeled RFS $\mathbf{X}$ (distributed according to $\boldsymbol{\pi}$ ) is distributed according to the marginal

$$
\begin{gathered}
\omega\left(\left\{\ell_{1}, \cdots, \ell_{n}\right\}\right) \triangleq \\
\left\{\begin{array}{cl}
\int \boldsymbol{\pi}\left(\left\{\left(x_{1}, \ell_{1}\right), \cdots,\left(x_{n}, \ell_{n}\right)\right\}\right) d\left(x_{1}, \cdots, x_{n}\right), & \text { if } n>0 \\
\boldsymbol{\pi}(\emptyset), & \text { if } n=0 .
\end{array}\right.
\end{gathered}
$$

The quantity $\omega\left(\left\{\ell_{1}, \cdots, \ell_{n}\right\}\right)$ is referred to as the joint existence probability of the label set $\left\{\ell_{1}, \cdots, \ell_{n}\right\}$ in this paper. 
Definition 1. Given an LMO density $\pi$ on $\mathcal{F}(\mathbb{X} \times \mathbb{L})$, we define a function $P(\mathbf{X})$ on $\mathcal{F}(\mathbb{X} \times \mathbb{L})$ as (4).

Remark 1. Given a certain set of distinct labels $\left\{\alpha_{1}, \cdots, \alpha_{n}\right\}$, if $n>0$ and the weight $\omega\left(\left\{\alpha_{1}, \cdots, \alpha_{n}\right\}\right)>0$, $P\left(\left\{\left(x_{1}, \alpha_{1}\right), \cdots,\left(x_{n}, \alpha_{n}\right)\right\}\right)$ is essentially a joint probability density on $\mathbb{X}^{n}$ conditional on their corresponding labels $\alpha_{1}, \cdots, \alpha_{n}$. Indeed, from Definition 1] the LMO density $\boldsymbol{\pi}$ can be decomposed as

$$
\boldsymbol{\pi}(\mathbf{X})=\omega(\mathcal{L}(\mathbf{X})) P(\mathbf{X}) .
$$

2) Common Labeled RFSs: The most commonly used labeled RFSs in existing labeled multi-object filtering algorithms belong to the GLMB RFS family [14] [15]. They are distributed according to

$$
\boldsymbol{\pi}(\mathbf{X})=\Delta(\mathbf{X}) \sum_{c \in \mathbb{C}} \omega^{(c)}(\mathcal{L}(\mathbf{X}))\left[p^{(c)}\right]^{\mathbf{X}}
$$

where $\mathbb{C}$ is a discrete space, each $p^{(c)}(\cdot, \ell)$ is a probability density, and each $w^{(c)}(I)$ is non-negative with $\sum_{(I, c) \in \mathcal{F}(\mathbb{L}) \times \mathbb{C}} w^{(c)}(I)=1$.

The class of LMB RFSs is a subclass of the GLMB RFS family. An LMB RFS with state space $\mathbb{X}$ and label space $\mathbb{L}$ is distributed according to [14], [15], [18]

$$
\boldsymbol{\pi}(\mathbf{X})=\Delta(\mathbf{X}) \omega(\mathcal{L}(\mathbf{X})) p^{\mathbf{X}}
$$

where

$$
\omega(L)=\prod_{i \in \mathbb{L}}\left(1-r^{(i)}\right) \prod_{\ell \in L} \frac{1_{\mathbb{L}}(\ell) r^{(\ell)}}{1-r^{(\ell)}}
$$

and $r^{(\ell)}$ represents the existence probability of track $\ell$, and $p(\cdot, \ell)$ is the probability density of the kinematic state of track $\ell$ given its existence.

From (7) and (8), an LMB RFS is completely determined by the parameters $r^{(\ell)}$ for each $\ell \in \mathbb{L}$ and a function $p(x, \ell)$ defined on $\mathbb{X} \times \mathbb{L}$. Also, an LMB RFS can be completely characterized by its LMB parameters, i.e.,

$$
\boldsymbol{\pi}=\left\{\left(r^{(\alpha)}, p^{(\alpha)}(x, \ell)\right): \alpha \in \mathbb{L}\right\},
$$

with

$$
p^{(\alpha)}(x, \ell) \triangleq \delta_{\alpha}(\ell) p(x, \ell)
$$

Note that the definition in $(10)$ is applied for all the LMB RFSs throughout the paper.

Definition 2. Given the LMB parameters $\left\{\left(r^{(\alpha)}, p^{(\alpha)}(\cdot)\right)\right\}_{\alpha \in \mathbb{L} \text {, }}$ the labeled Bernoulli component $\left(r^{(\alpha)}, p^{(\alpha)}(\cdot)\right)$ is referred to as track $\alpha$, with $p^{(\alpha)}(\cdot)$ representing the joint spatial and label density, and $r^{(\alpha)}$ the probability of existence of track $\alpha$.

\section{Multi-object Bayes Filter}

Multi-object Bayes filter is at the core of multi-object filtering in RFS framework. This subsection provides a review of the multi-object Bayes filter in the formulation of labeled multi-object state, which is firstly presented in [14]. To incorporate object tracks, objects are identified by an ordered pair of integers $\ell=(k, i)$, where $k$ is the time of birth, and $i \in \mathbb{N}$ is a unique index to distinguish objects born at the same time. The label space for objects born at time $k$, denoted as $\mathbb{L}_{k}$, is then $\{k\} \times \mathbb{N}$. An object born at time $k$ has a state $\mathbf{x} \in \mathbb{X} \times \mathbb{L}_{k}$. The label space for objects at time $k$ (including those born prior to $k$ ), denoted as $\mathbb{L}_{0: k}$, is constructed recursively by $\mathbb{L}_{0: k}=\mathbb{L}_{0: k-1} \cup \mathbb{L}_{k}$. A multi-object state $\mathbf{X}$ at time $k$, is a finite subset of $\mathbb{X} \times \mathbb{L}_{0: k}$. Note that $\mathbb{L}_{0: k-1}$ and $\mathbb{L}_{k}$ are disjoint.

The multi-object posterior density $\boldsymbol{\pi}_{k}$ is propagated forward recursively by the multi-object Bayes filter,

$$
\begin{gathered}
\boldsymbol{\pi}_{k \mid k-1}\left(\mathbf{X}_{k}\right)=\int \mathbf{f}_{k \mid k-1}\left(\mathbf{X}_{k} \mid \mathbf{X}\right) \boldsymbol{\pi}_{k-1}(\mathbf{X}) \delta \mathbf{X} \\
\boldsymbol{\pi}_{k}\left(\mathbf{X}_{k}\right)=\frac{g_{k}\left(\Upsilon_{k} \mid \mathbf{X}_{k}\right) \boldsymbol{\pi}_{k \mid k-1}\left(\mathbf{X}_{k}\right)}{\int g_{k}\left(\Upsilon_{k} \mid \mathbf{X}\right) \boldsymbol{\pi}_{k \mid k-1}(\mathbf{X}) \delta \mathbf{X}}
\end{gathered}
$$

where $\boldsymbol{\pi}_{k \mid k-1}$ is the multi-object predicted density from time $k-1$ to time $k ; \mathbf{f}_{k \mid k-1}(\cdot \mid \cdot)$ is the multi-object transition density; $g_{k}\left(\Upsilon_{k} \mid \cdot\right)$ is the multi-object likelihood function and $\Upsilon_{k}$ denotes the observations of multi-object state at time $k$. Note that $\Upsilon_{k}$ is a general notation which can represent a vector observation $\mathrm{z}_{k}$, or a set observation $Z_{k}$, depending on the observation model adopted.

For convenience, in what follows we omit explicit references to the time index $k$, and denote $\mathbb{L} \triangleq \mathbb{L}_{0: k}, \mathbb{B} \triangleq \mathbb{L}_{k+1}, \mathbb{L}_{+} \triangleq$ $\mathbb{L} \cup \mathbb{B}, \boldsymbol{\pi} \triangleq \boldsymbol{\pi}_{k}, \boldsymbol{\pi}_{+} \triangleq \boldsymbol{\pi}_{k+1 \mid k}, g \triangleq g_{k}$, and $\mathbf{f} \triangleq \mathbf{f}_{k \mid k+1}$.

\section{Multi-object Transition Kernel}

This paper considers the standard multi-object transition model [1], [14]. Given a labeled multi-object state $\mathbf{X}$, each state $(x, \ell) \in \mathbf{X}$ either continues to exist at the next time step with probability $p_{S}(x, \ell)$ and evolves to a new state $\left(x_{+}, \ell_{+}\right)$ with probability density $f_{+}\left(x_{+}, \ell_{+} \mid x, \ell\right)=f_{+}\left(x_{+} \mid x, \ell\right) \delta_{\ell_{+}}(\ell)$, or dies with probability $1-p_{S}(x, \ell)$. According to Definition 1 . The set of new objects born at the next time step defined on $\mathbb{X} \times \mathbb{B}$ is distributed according to

$$
\mathbf{f}_{B}(\mathbf{Y})=\omega_{B}(\mathcal{L}(\mathbf{Y})) P_{B}(\mathbf{Y})
$$

Note that the birth density $\mathbf{f}_{B}$ also can be specified as an LMB density of form (7) or a GLMB density of form (6).

A multi-object state $\mathbf{X}_{+}$is the superposition of surviving objects and newly born objects. Assuming that the surviving and the newly born object states evolve independently, the multi-object transition function is given by [15]

$$
\mathbf{f}\left(\mathbf{X}_{+} \mid \mathbf{X}\right)=\mathbf{f}_{S}\left(\mathbf{X}_{+} \cap(\mathbb{X} \times \mathbb{L}) \mid \mathbf{X}\right) \mathbf{f}_{B}\left(\mathbf{X}_{+} \cap(\mathbb{X} \times \mathbb{B})\right)
$$

where

$$
\begin{gathered}
\mathbf{f}_{S}(\mathbf{W} \mid \mathbf{X})=\Delta(\mathbf{W}) \Delta(\mathbf{X}) 1_{\mathcal{L}(\mathbf{X})}(\mathcal{L}(\mathbf{W}))[\Phi(\mathbf{W} ; \cdot)]^{\mathbf{X}} \\
\Phi(\mathbf{W} ; x, \ell)= \begin{cases}p_{S}(x, \ell) \delta_{\ell_{+}}(\ell) f_{+}\left(x_{+} \mid x, \ell\right) & \text { if }\left(x_{+}, \ell\right) \in \mathbf{W} \\
1-p_{S}(x, \ell), & \text { if } \ell \notin \mathcal{L}(\mathbf{W}) .\end{cases}
\end{gathered}
$$

\section{E. Generic Observation Model}

The standard formulation in the RFS based multi-object tracking is based on the standard observation model [6] where observation data is assumed to have been preprocessed into thresholded detections, each object is assumed to cause at most one detection, and each detection is assumed to be either a false alarm (clutter) or generated from one object. The tracking filters under the standard observation model have been well investigated. One remarkable development is the 


$$
P\left(\left\{\left(x_{1}, \ell_{1}\right), \cdots,\left(x_{n}, \ell_{n}\right)\right\}\right) \triangleq\left\{\begin{array}{cl}
\frac{\pi\left(\left\{\left(x_{1}, \ell_{1}\right), \cdots,\left(x_{n}, \ell_{n}\right)\right\}\right)}{\omega\left(\left\{\ell_{1}, \cdots, \ell_{n}\right\}\right)}, & \text { if } n>0 \text { and } w\left(\left\{\ell_{1}, \cdots, \ell_{n}\right\}\right)>0 \\
1, & \text { if } n>0 \text { and } w\left(\left\{\ell_{1}, \cdots, \ell_{n}\right\}\right)=0 \\
1, & \text { if } n=0 .
\end{array}\right.
$$

GLMB family of densities are conjugate prior with respect to the standard multi-object likelihood. Utilizing this property, the GLMB filter is proposed as a closed form of the multiobject Bayes filter under the standard observation model, and its performance has been well demonstrated in [14], [15].

This paper considers the generic observation model which has the indication that no simplifying assumptions on the multi-object likelihood are made. The terminology "generic measurement (observation) model" first arised in [19] for the $\delta$-GLMB filter with the generic multi-object likelihood.

The considered GOM covers both the standard and nonstandard observation models. As the first category of sensor models has been well investigated, this paper mainly focuses on the non-standard sensor models. Below, we present two typical examples for the non-standard observation models, namely, the pixled TBD model and the acoustic amplitude sensor model.

Example 1 - Pixeled TBD Model: The surveillance region is divided into $M$ cells. The observations at the current time step are collected in the vector $\mathrm{z}=\left(z_{1}, \cdots, z_{M}\right) \in \mathbb{R}^{M}$, with $z_{j}$ being the intensity observation obtained in the $j$ th cell. An object $\mathrm{x}$ can illuminate several cells of its surroundings. Within the effective template of $\mathbf{x}$, the intensity contribution from $\mathbf{x}$ to the $j$ th cell follows a point spread function [36]

$$
c_{j}(\mathbf{x})=\frac{\delta_{x} \delta_{y} \sigma_{T}}{2 \pi \sigma_{b}^{2}} \exp \left(-\frac{\left(\delta_{x} a-p_{x}\right)^{2}+\left(\delta_{y} b-p_{y}\right)^{2}}{2 \sigma_{b}^{2}}\right)
$$

where $\sigma_{T}$ is the source intensity, $\sigma_{b}^{2}$ is the blurring factor, $\delta_{x}$ and $\delta_{y}$ are the cell side lengths, and $j=(a, b)$ denotes the position of the $j$ th cell in a two-dimensional image of the surveillance region. For the cells beyond the effective template of $\mathbf{x}$, the intensity contribution $c_{j}(\mathbf{x})=0$.

The observations obtained from different cells are assumed to be independently distributed conditioned on the multi-object state $\mathbf{X}$, and thus the multi-object likelihood is

$$
g(\mathrm{z} \mid \mathbf{X})=\prod_{j=1}^{M} g\left(z_{j} \mid \mathbf{X}\right)
$$

where $g\left(z_{j} \mid \mathbf{X}\right)$ denotes the likelihood of the $j$ th cell. The distribution of $g\left(z_{j} \mid \mathbf{X}\right)$ varies from different applications. For instance, in the infrared/image application [13], [36], the likelihood $g\left(z_{j} \mid \mathbf{X}\right)$ is assumed to be a Gaussian distribution,

$$
g\left(z_{j} \mid \mathbf{X}\right)=\mathcal{N}\left(z_{j} ; \sum_{\mathbf{x} \in \mathbf{X}} c_{j}(\mathbf{x}), \sigma_{N}^{2}\right)
$$

where $\mathcal{N}(z ; \mu, \Gamma)$ denotes the Gaussian probability density evaluated at $z$ with mean $\mu$ and covariance matrix $\Gamma$.

Example 2 - Acoustic Amplitude Sensor: We consider a wireless sensor network consisting of $\mathcal{M}$ sensors [33]. At a certain time step, sensor $m(m \in\{1, \cdots, \mathcal{M}\})$ acquires an observation $z_{m} \in \mathbb{R}$. Each object $\mathbf{x}$ emits a sound with amplitude $A$ that is assumed to be constant. For sensor $m$ located at position $\xi_{m}$, the received sound amplitude due to target $\mathbf{x}$ is modelled as $A /\left\|\rho(\mathbf{x})-\xi_{m}\right\|^{\kappa}$, where $\rho(\mathbf{x})$ is the position of object $\mathbf{x}$, and $\kappa$ is the path loss exponent. The scalar observation $z_{m}$ obtained by sensor $m$ is then given by

$$
z_{m}=h_{m}(\mathbf{X})+v_{m}
$$

with

$$
h_{m}(\mathbf{X})=\sum_{\mathbf{x} \in \mathbf{X}} \frac{A}{\left\|\rho(\mathbf{x})-\xi_{m}\right\|^{\kappa}}
$$

where $v_{m} \sim \mathcal{N}\left(0, \sigma_{v}^{2}\right)$ for $m=1, \cdots, \mathcal{M}$ are zero-mean Gaussian noise variables of equal variance $\sigma_{v}^{2}$. Assume that $v_{1}, \cdots, v_{\mathcal{M}}$ are mutually independent and independent of $\mathbf{X}$. The likelihood function between observation vector $\mathrm{z}=$ $\left(z_{1} \cdots, z_{\mathcal{M}}\right) \in \mathbb{R}^{\mathcal{M}}$ and multi-object state $\mathbf{X}$ is given by

$$
g(\mathbf{z} \mid \mathbf{X})=\prod_{m=1}^{\mathcal{M}} \mathcal{N}\left(z_{m} ; h_{m}(\mathbf{X}), \sigma_{v}^{2}\right) .
$$

Note that both (19) and (22) are highly non-linear likelihoods which are not closed under the Bayes update equation (12).

\section{THE LMO-GOM FILTER}

In this section, we derive the multi-object prediction and update equations of Bayes filter by specifying the multiobject prior and posterior as the product styled LMO densities provided in (5). The result is an exact solution for labeled multi-object Bayes filter with GOM under the standard multiobject transition model, and thus is called as the LMO-GOM filter. Furthermore, an SMC implementation of the LMOGOM filter is presented.

\section{A. Recursive Equations of the LMO-GOM Filter}

Proposition 1. Suppose that the current multi-object prior density is a general LMO density of the form (5) and the birth density is also a general LMO density of the form (13), then the multi-object predicted density under the multi-object transition function of the form (14) is given by

$$
\boldsymbol{\pi}_{+}\left(\mathbf{X}_{+}\right)=\omega_{+}\left(\mathcal{L}\left(\mathbf{X}_{+}\right)\right) P_{+}\left(\mathbf{X}_{+}\right)
$$

on state space $\mathbb{X}$ and label space $\mathbb{L}_{+}=\mathbb{L} \cup \mathbb{B}$, where

$$
\begin{aligned}
\omega_{+}(L) & =\omega_{B}(L \cap \mathbb{B}) \omega_{S}(L \cap \mathbb{L}) \\
P_{+}\left(\mathbf{X}_{+}\right) & =P_{B}\left(\mathbf{X}_{+} \cap \mathbb{X} \times \mathbb{B}\right) P_{S}\left(\mathbf{X}_{+} \cap \mathbb{X} \times \mathbb{L}\right) \\
\omega_{S}(J) & =\sum_{I \subseteq \mathbb{L}} 1_{I}(J) \omega(I) \eta_{S, I}(J) \\
P_{S}(\mathbf{W}) & =\frac{\sum_{I \subseteq \mathbb{L}} 1_{I}(\mathcal{L}(\mathbf{W})) \omega(I) P_{S, I}(\mathbf{W})}{\omega_{S}(\mathcal{L}(\mathbf{W}))}
\end{aligned}
$$

with

$$
\begin{aligned}
P_{S,\left\{\ell_{1}, \cdots, \ell_{n}\right\}}(\mathbf{W}) & =\int 1_{\left\{\ell_{1}, \cdots, \ell_{n}\right\}}(\mathcal{L}(\mathbf{W})) \prod_{i=1}^{n} \Phi\left(\mathbf{W} ; x_{i}, \ell_{i}\right) \\
& \cdot P\left(\left\{\left(x_{1}, \ell_{1}\right) \cdots,\left(x_{n}, \ell_{n}\right)\right\}\right) d\left(x_{1} \cdots x_{n}\right)
\end{aligned}
$$




$$
\begin{aligned}
& \eta_{S,\left\{\ell_{1}, \cdots, \ell_{n}\right\}}\left(\left\{\ell_{+, 1}, \cdots, \ell_{+, n_{+}}\right\}\right)=\int P_{S,\left\{\ell_{1}, \cdots, \ell_{n}\right\}} . \\
& \quad\left(\left\{\left(x_{+, 1}, \ell_{+, 1}\right), \cdots,\left(x_{+, n_{+}}, \ell_{+, n_{+}}\right)\right\}\right) d\left(x_{+, 1}, \cdots, x_{+, n_{+}}\right) .
\end{aligned}
$$

Proof. See Appendix A.

Proposition 1 explicitly describes how to calculate $\omega_{+}(\cdot)$ and $P_{+}(\cdot)$ of the multi-object predicted density from $\omega(\cdot)$ and $P(\cdot)$ of the multi-object prior density. We note that $\boldsymbol{\pi}_{S}(\mathbf{W})=\omega_{S}(\mathcal{L}(\mathbf{W})) P_{S}(\mathbf{W})$ is the density of the surviving objects with $\omega_{S}(\cdot)$ and $P_{S}(\cdot)$ shown in $(26)$ and $(27)$. For a given label set $J, \omega_{S}(J)$ is the weighted sum of the prior weights $\omega(I)$ over all subsets of $\mathbb{L}$ that contain the surviving set $J$. The function $P_{S}(\mathbf{W})$ given a certain label set $\mathcal{L}(\mathbf{W})$ is also a weighted sum of $P_{S, I}(\mathbf{W})$ terms over all subsets of $\mathbb{L}$ that contain the surviving set $\mathcal{L}(\mathbf{W})$. $P_{S,\left\{\ell_{1}, \cdots, \ell_{n}\right\}}(\mathbf{W})$ in 28 for a certain $\mathcal{L}(\mathbf{W})$ is a nonnormalized joint density evolved from the prior joint probability density $P\left(\left\{\left(x_{1}, \ell_{1}\right), \cdots,\left(x_{n}, \ell_{n}\right)\right\}\right)$ with the "pseudo" transition density $\prod_{i=1}^{n} \Phi\left(\mathbf{W} ; x_{i}, \ell_{i}\right) . P_{S,\left\{\ell_{1}, \cdots, \ell_{n}\right\}}(\mathbf{W})$ is conditional on that the previous label set is $I=\left\{\ell_{1}, \cdots, \ell_{n}\right\}$ and only the objects with the label set $\mathcal{L}(\mathbf{W}) \subseteq I$ exist after evolving. $\eta_{S,\left\{\ell_{1}, \cdots, \ell_{n}\right\}}\left(I_{+}\right)$in $\sqrt{29}$ is essentially the normalizing constant of $P_{S,\left\{\ell_{1}, \cdots, \ell_{n}\right\}}(\mathbf{W})$ with $\mathcal{L}(\mathbf{W})=I_{+}$.

Utilizing the independence of surviving objects and newly born objects, the multi-object predicted density can be obtained by multiplying the weights and the corresponding joint probability densities of newly born objects and surviving objects.

Proposition 2. Suppose that the current multi-object predicted density is a general LMO density of the form (23), then the multi-object posterior density under a generic multi-object likelihood $g(Z \mid \mathbf{X})$ is given by

$$
\boldsymbol{\pi}(\mathbf{X} \mid \Upsilon)=\omega(\mathcal{L}(\mathbf{X}) ; \Upsilon) P(\mathbf{X} ; \Upsilon)
$$

on state space $\mathbb{X}$ and the label space $\mathbb{L}_{+}$, where

$$
\begin{aligned}
P(\mathbf{X} ; \Upsilon) & =\frac{g(\Upsilon \mid \mathbf{X}) P_{+}(\mathbf{X})}{\eta_{\Upsilon}(\mathcal{L}(\mathbf{X}))} \\
\omega\left(I_{+} ; \Upsilon\right) & =\frac{\eta_{\Upsilon}\left(I_{+}\right) \omega_{+}\left(I_{+}\right)}{\sum_{I_{+} \in \mathbb{L}_{+}} \eta_{\Upsilon}(I) \omega_{+}(I)}
\end{aligned}
$$

with

$$
\begin{array}{r}
\eta \Upsilon\left(\left\{\ell_{1}, \ell_{2}, \cdots, \ell_{n}\right\}\right)=\int g\left(\Upsilon \mid\left\{\left(x_{1}, \ell_{1}\right), \cdots,\left(x_{n}, \ell_{n}\right)\right\}\right) \\
\cdot P_{+}\left(\left\{\left(x_{1}, \ell_{1}\right), \cdots,\left(x_{n}, \ell_{n}\right)\right\}\right) d\left(x_{1}, \cdots, x_{n}\right) .
\end{array}
$$

\section{Proof. See Appendix B.}

Proposition 2 explicitly describes how to calculate the parameters $\omega(\cdot ; \Upsilon)$ and $P(\cdot \mid \Upsilon)$ of the multi-object posterior density from the parameters $\omega_{+}(\cdot)$ and $P_{+}(\cdot)$ of the multiobject predicted density. For a given label set $\mathcal{L}(\mathbf{X})$, the posterior joint probability density $P(\mathbf{X} \mid \Upsilon)$ in 31 is computed from the prior joint probability density $P_{+}(\overline{\mathbf{X}})$ via "Bayes' rule" with likelihood $g(\Upsilon \mid \mathbf{X})$. For a given label set $I$, the posterior weight $\omega(I ; \Upsilon)$ is proportional to the predicted weight $\omega_{+}(I)$ scaled by the normalizing constant $\eta_{\Upsilon}(I)$.

\section{B. The SMC Implementation of the LMO-GOM Filter}

In the above subsection, the combination of Propositions 1 and 2 provides an exact Bayesian solution by adopting the decomposition of the LMO density in the form of (5). Hence, an intuitive implementation of the LMO-GOM filter is to recursively compute the functions $\omega(\cdot)$ and $P(\cdot)$ at each time step. However, when implementing the LMO-GOM filter, the approximation of $P(\mathbf{X})$ is not straightforward since $P(\mathbf{X})$ (defined on $\mathcal{F}(\mathbb{X} \times \mathbb{L})$ ) is not a probability density. To this end, we represent the product styled of the LMO density of the form (5) in another equivalent form as Remark 2 .

Remark 2. An LMO density $\pi$ on $\mathcal{F}(\mathbb{X} \times \mathbb{L})$ can be expressed as a mixture of multi-object densities,

$$
\boldsymbol{\pi}(\mathbf{X})=\sum_{I \in \mathcal{F}(\mathbb{L})} \omega^{(I)} P^{(I)}(\mathbf{X})
$$

where

$$
\begin{aligned}
\omega^{(I)} & \triangleq \omega(I), \\
P^{(I)}(\mathbf{X}) & \triangleq \delta_{I}(\mathcal{L}(\mathbf{X})) P(\mathbf{X})
\end{aligned}
$$

in which the definitions of $P(\mathbf{X})$ and $\omega(I)$ are given in Definition $1, \omega^{(I)}$ denotes the existence probability of the label set I satisfying $\sum_{I \in \mathcal{F}(\mathbb{L})} \omega^{(I)}=1$, and $P^{(I)}(\cdot)$ is the multiobject probability density (defined on $\mathcal{F}(\mathbb{X} \times \mathbb{L})$ ) conditional on the existence of the label set I. Hence, $\boldsymbol{\pi}(\cdot)$ is completely characterized by a set of parameters $\left\{\left(\omega^{(I)}, P^{(I)}(\cdot)\right)\right\}_{I \in \mathcal{F}(\mathbb{L}) \text {. }}$.

The integrals of $P^{(I)}(\mathbf{X})$ and $P(\mathbf{X})$ have the following relationship. For any $I=\left\{\alpha_{1}, \cdots, \alpha_{n}\right\} \in \mathcal{F}(\mathbb{L})$, and given an arbitrary function $v(\mathbf{X})$ on $\mathcal{F}(\mathbb{X} \times \mathbb{L})$, we have

$$
\begin{aligned}
& \int P^{(I)}(\mathbf{X}) v(\mathbf{X}) \delta \mathbf{X}=\int \delta_{I}(\mathcal{L}(\mathbf{X})) P(\mathbf{X}) v(\mathbf{X}) \delta \mathbf{X} \\
= & \int P\left(\left\{\left(x_{1}, \alpha_{1}\right), \cdots,\left(x_{n}, \alpha_{n}\right)\right\}\right) \\
& v\left(\left\{\left(x_{1}, \alpha_{1}\right), \cdots,\left(x_{n}, \alpha_{n}\right)\right\}\right) d\left(x_{1}, \cdots, x_{n}\right) .
\end{aligned}
$$

Eq. 37 indicates that the set integral of $P^{(I)}(\cdot)$ is equivalent to the Euclidean notion of integral of the joint probability density $P\left(\left\{\left(\cdot, \alpha_{1}\right), \cdots,\left(\cdot, \alpha_{n}\right)\right\}\right)$ on $\mathbb{X}^{|I|}$.

Utilizing the formulas provided in Remark 2, implementing the LMO-GOM filter, based on Propositions 1 and 2, amounts to computing the predicted parameter set $\left\{\left(\omega_{+}^{\left(I_{+}\right)}, P_{+}^{\left(I_{+}\right)}(\cdot)\right)\right\}_{I_{+} \in \mathcal{F}\left(\mathbb{L}_{+}\right)}$with $\mathbb{L}_{+}=\mathbb{L} \cup \mathbb{B}$ and

$$
\omega_{+}^{\left(I_{+}\right)}=\omega_{+}\left(I_{+}\right) ; P_{+}^{\left(I_{+}\right)}\left(\mathbf{X}_{+}\right)=\delta_{I_{+}}\left(\mathcal{L}\left(\mathbf{X}_{+}\right)\right) P_{+}\left(\mathbf{X}_{+}\right),
$$

and the posterior parameter set $\left\{\left(\omega^{\left(I_{+}\right)}(\Upsilon), P^{\left(I_{+}\right)}(\cdot ; \Upsilon)\right)\right\}_{I_{+} \in \mathcal{F}\left(\mathbb{L}_{+}\right)}$with

$\omega^{\left(I_{+}\right)}(\Upsilon)=\omega\left(I_{+} ; \Upsilon\right) ; P^{\left(I_{+}\right)}(\mathbf{X} ; \Upsilon)=\delta_{I_{+}}(\mathcal{L}(\mathbf{X})) P(\mathbf{X} ; \Upsilon)$

forwards in time.

As it was mentioned earlier, our algorithms are mainly designed for the non-standard observation model which usually involves the non-Gaussian/non-linear model and has no closedfrom solution. Hence, in this subsection, we provide an SMC implementation of the LMO-GOM filter. Each $P^{(I)}(\mathbf{X})$ is represented by a set of weighted particles. Associated weights, and normalizing constants can be computed from particles and their weights. 
Suppose that the current prior parameter set is $\boldsymbol{\pi}=$ $\left\{\left(\omega^{(I)}, P^{(I)}(\cdot)\right)\right\}_{I \in \mathcal{F}(\mathbb{L})}$ where each $P^{(I)}(\mathbf{X})$ is approximated with a set of particles $\left\{\left(w_{j}^{(I)}, \mathbf{X}_{j}^{(I)}\right)\right\}_{j=1}^{N_{p}^{(I)}}$, i.e.,

$$
P^{(I)}(\mathbf{X})=\sum_{j=1}^{N_{p}^{(I)}} w_{j}^{(I)} \delta_{\mathbf{X}_{j}^{(I)}}(\mathbf{X}) .
$$

Utilizing (37), the quantities in the prediction step are computed as

$$
\begin{gathered}
P_{S, I}(\mathbf{W})=\int 1_{I}(\mathcal{L}(\mathbf{W}))[\Phi(\mathbf{W} ; \cdot)]^{\mathbf{X}} P^{(I)}(\mathbf{X}) \delta \mathbf{X} \\
=\sum_{j=1}^{N_{p}^{(I)}} 1_{I}(\mathcal{L}(\mathbf{W})) w_{j}^{(I)}[\Phi(\mathbf{W} ; \cdot)]^{\mathbf{X}_{j}^{(I)},} \\
\eta_{S, I}(J)=\int \delta_{J}(\mathcal{L}(\mathbf{W})) P_{S, I}(\mathbf{W}) \delta \mathbf{W} \\
=\sum_{j=1}^{N_{p}^{(I)}} w_{j}^{(I)}\left[p_{S}\right]^{\mathbf{X}_{j}^{(I)} \cap \mathbb{X} \times J}\left[1-p_{S}\right]^{\mathbf{X}_{j}^{(I)} \cap \mathbb{X} \times(I-J) .}
\end{gathered}
$$

Then $\omega_{S}(J)$ is computed by substitution of 40 into 26 , and $\omega_{+}(L)$ is computed by substitution of the computed $\omega_{S}(J)$ into (24).

For each label set $I_{+} \in \mathcal{F}\left(\mathbb{L}_{+}\right)$, firstly, choose a subset $\mathbb{U}$ of $\mathcal{F}(\mathbb{L})$ according to

$$
\mathbb{U}=\left\{I \in \mathcal{F}(\mathbb{L}): 1_{I}\left(I_{+} \cap \mathbb{L}\right)=1\right\} .
$$

Utilizing 25, 27) and 31, the posterior parameter $P^{\left(I_{+}\right)}(\cdot ; \Upsilon)$ is computed as

$$
\begin{aligned}
& P_{+}^{\left(I_{+}\right)}(\mathbf{X} ; \Upsilon) \propto g(\Upsilon \mid \mathbf{X}) \delta_{I_{+} \cap \mathbb{B}}\left(\mathcal{L}\left(\mathbf{X}_{B}\right)\right) P_{B}\left(\mathbf{X}_{B}\right) \times \\
& \delta_{I_{+} \cap \mathbb{L}}\left(\mathcal{L}\left(\mathbf{X}_{S}\right)\right) \sum_{I \in \mathbb{U}} \bar{\omega}^{(I)} \sum_{j=1}^{N_{p}^{(I)}} w_{j}^{(I)}\left[\Phi\left(\mathbf{X}_{S} ; \cdot\right)\right]^{\mathbf{X}_{j}^{(I)}}
\end{aligned}
$$

where

$$
\begin{gathered}
\mathbf{X}_{S} \triangleq \mathbf{X}_{+} \cap \mathbb{X} \times \mathbb{L}, \mathbf{X}_{B} \triangleq \mathbf{X}_{+} \cap \mathbb{X} \times \mathbb{B} \\
\bar{\omega}^{(I)}=\frac{\omega^{(I)}}{\omega_{S}\left(I_{+} \cap \mathbb{L}\right)} .
\end{gathered}
$$

By employing the idea of the auxiliary particle filter [23][25], sampling from (41) can be achieved by sampling from the higher dimensional joint density

$$
\begin{array}{r}
P_{+}^{\left(I_{+}\right)}(\mathbf{X}, \mathcal{I}, u ; \Upsilon) \propto g(\Upsilon \mid \mathbf{X}) \delta_{I_{+} \cap \mathbb{B}}\left(\mathcal{L}\left(\mathbf{X}_{B}\right)\right) P_{B}\left(\mathbf{X}_{B}\right) \\
\delta_{I_{+} \cap \mathbb{L}}\left(\mathcal{L}\left(\mathbf{X}_{S}\right)\right) \bar{\omega}^{(\mathcal{I})} w_{u}^{(\mathcal{I})}\left[\Phi\left(\mathbf{X}_{S} ; \cdot\right)\right]^{\mathbf{X}_{u}^{(\mathcal{I})}} .
\end{array}
$$

where the auxiliary variable $\mathcal{I} \in \mathbb{U}$ is the previous label set from which the current label set $I_{+}$is evolved, and the auxiliary variable $u \in\left\{1, \cdots, N_{p}^{(\mathcal{I})}\right\}$ is the index on the sample at the previous time step conditional on the previous label set $\mathcal{I}$. The auxiliary variables aid in the sampling of suitable values of the multi-target state $\mathbf{X}$. They are discarded after the sampling procedure is completed. States $\mathbf{X}_{j_{+}}^{\left(I_{+}\right)}$, the previous label set $\mathcal{I}_{j_{+}}$, and particle indices $u_{j_{+}}$are drawn from an importance density $q^{\left(I_{+}\right)}(\mathbf{X}, \mathcal{I}, u \mid \Upsilon)$ for $j_{+}=1, \cdots, N_{p}^{\left(I_{+}\right)}$, and the un-normalized weight is computed as

$$
\begin{aligned}
& \widetilde{w}_{j_{+}}^{\left(I_{+}\right)}=g(\Upsilon \mid \mathbf{X}) \delta_{I_{+} \cap \mathbb{B}}\left(\mathcal{L}\left(\mathbf{X}_{B}\right)\right) P_{B}\left(\mathbf{X}_{B}\right) \bar{\omega}^{(\mathcal{I})} \\
& \delta_{I_{+} \cap \mathbb{L}}\left(\mathcal{L}\left(\mathbf{X}_{S}\right)\right) w_{u}^{(\mathcal{I})}\left[\Phi\left(\mathbf{X}_{S} ; \cdot\right)\right]^{\mathbf{X}_{u}^{(\mathcal{I})}} / q\left(\mathbf{X}_{j_{+}}^{\left(I_{+}\right)}, \mathcal{I}_{j_{+}}, u_{j_{+}}\right)
\end{aligned}
$$

A feasible choice of the proposal function $q^{\left(I_{+}\right)}(\mathbf{X}, \mathcal{I}, u \mid \Upsilon)$ is as follows:

$$
\begin{aligned}
q^{\left(I_{+}\right)}(\mathbf{X}, \mathcal{I}, u \mid \Upsilon) & =\delta_{I_{+} \cap \mathbb{B}}\left(\mathcal{L}\left(\mathbf{X}_{B}\right)\right) P_{B}\left(\mathbf{X}_{B}\right) \\
\cdot & \delta_{I_{+} \cap \mathbb{L}}\left(\mathcal{L}\left(\mathbf{X}_{S}\right)\right) \bar{\omega}^{(\mathcal{I})} w_{u}^{(\mathcal{I})}\left[\Phi\left(\mathbf{X}_{S} ; \cdot\right)\right]^{\mathbf{X}_{u}^{(\mathcal{I})}}
\end{aligned}
$$

In this case, the un-normalized weight is computed as

$$
\widetilde{w}_{j_{+}}^{\left(I_{+}\right)}=g\left(\Upsilon \mid \mathbf{X}_{j_{+}}^{\left(I_{+}\right)}\right) .
$$

Note that it is possible to design a more sophisticated proposal density than (46), but it is beyond the scope of this paper.

Utilizing 37), the quantity $\eta_{\Upsilon}\left(I_{+}\right)$is computed as

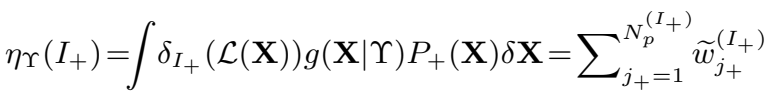

and the posterior parameter $\omega^{\left(I_{+}\right)}(\Upsilon)=\omega\left(I_{+} ; \Upsilon\right)$ is computed by substitution of (47) into (32).

Resampling and Implementation Issues: After the update step, for each $P^{\left(I_{+}\right)}(\cdot ; \Upsilon)$, perform resampling [37] to obtain an evenly weighted particle set. To reduce the growing number of parameters, the pair of posterior parameters $\left(w^{\left(I_{+}\right)}(\Upsilon), P^{\left(I_{+}\right)}(\cdot ; \Upsilon)\right)$ with existence probabilities $w^{\left(I_{+}\right)}(\Upsilon)$ below a threshold are discarded [14], [16], [19].

\section{Discussions and Analysis}

The LMO-GOM filter provides an exact Bayesian solution for the labeled multi-object tracking problem under the GOM and the standard transition kernel. Nevertheless, in general, the LMO-GOM filter can be computationally prohibitive, especially for a large number of objects. Computing integrals on high-dimensional spaces (the integral of $P^{(I)}(\cdot)$ ) and exponential growth of the number of parameters with the number of objects are the two main reasons in many applications.

Observing Proposition 1, the prediction step of the LMOGOM filter can be further simplified for particular multiobject priors such as the $\delta$-GLMB and LMB densities. This can be achieved due to the independence assumption between object motions when formulating the multi-object transition kernel. The $\delta$-GLMB-GOM filter [19] is essentially derived by approximating the multi-object posterior as a principled $\delta$-GLMB density and assuming the $\delta$-GLMB prior.

Remark 3. The implementation of the LMO-GOM filter only involves one source of inaccuracy which is the numerical error caused by the Monte Carlo (MC) approximation of the highdimensional integral. Based on the convergence properties of the MC approximation [38], when the number of samples approaches infinity, the numerical errors of the integral computations approach zero, and the LMO-GOM filter is implemented with perfect accuracy. Hence, with sufficient computing resources, the LMO-GOM filter is expected to exhibit the optimal performance, and possibly served as a performance benchmark in labeled multi-object tracking with the standard transition kernel. In comparison, the implementation of the $\delta$ GLMB filter involves two sources of inaccuracy. One is related to the approximation of posteriors and the other to the $M C$ approximation. Due to the first source of inaccuracy, even if the number of particles approaches infinity, the $\delta$-GLMB filter is not exact. 


\section{THE LMB-GOM FILTER}

As an efficient approximation of the LMO-GOM filter, the $\delta$-GLMB-GOM filter alleviates the computation burden by simplifying the prediction equation. However, the $\delta$ GLMB prediction can be still intractable (both memory- and computational-wise) due to the exponential growth of the number of terms of multi-object exponentials in the predicted $\delta$-GLMB density with the number of objects.

In this section, we further explore more tractable approximations of the LMO-GOM filter. [18] proposed an LMB filter for the standard multi-object likelihood which approximates the $\delta$-GLMB multi-object posterior as a principled LMB density preserving the first-order moment, and further proposed a dynamic grouping procedure based implementation which drastically reduces execution time with slight accuracy promise by exploiting the mathematical formulation of the LMB prior. Motivated by the LMB filter and its fast implementation proposed in [18], in this section, we seek the "best" LMB approximation to replace the full multi-object posterior under the GOM, and consequently, develop an extension of the LMB filter for the GOM, referred to as the LMB-GOM filter. Furthermore, we also present an efficient implementation for the LMB-GOM filter based on a dynamic grouping procedure.

\section{A. The "Best" LMB Approximation}

In this subsection, we derive the "best" LMB approximation of the general LMO density. Herein, the "best" approximation means the best information-theoretic fit in terms of the minimal KLD. Propositions 3 and 4, respectively, derive the explicit formulas of the labeled PHDs for the general LMO density and the LMB density, which are the basis of the derivation of the "best" LMB approximation. Proposition 5 provides the explicit formula for the "best" LMB approximation of the general LMO density.

Proposition 3. Given an arbitrary LMO density $\boldsymbol{\pi}(\mathbf{X})=$ $\omega(\mathcal{L}(\mathbf{X})) P(\mathbf{X})$ on state space $\mathbb{X}$ and label space $\mathbb{L}$, the labeled PHD of $\pi$ is

$$
v(x, \ell)=\sum_{I \in \mathcal{F}(\mathbb{L})} 1_{I}(\ell) \omega(I) p_{I-\{\ell\}}(x, \ell)
$$

where “-” denotes set difference, and

$$
\begin{array}{r}
p_{\left\{\ell_{1}, \cdots, \ell_{n}\right\}}(x, \ell)=\int P\left(\left\{(x, \ell),\left(x_{1}, \ell_{1}\right), \cdots,\right.\right. \\
\left.\left.\left(x_{n}, \ell_{n}\right)\right\}\right) d\left(x_{1}, \cdots, x_{n}\right) .
\end{array}
$$

Proof. See Appendix C.

Proposition 4. Given an $L M B$ RFS with the $L M B$ parameters $\boldsymbol{\pi}=\left\{\left(r^{(\alpha)}, p^{(\alpha)}(\cdot)\right)\right\}_{\alpha \in \mathbb{L}}$, the labeled PHD of $\boldsymbol{\pi}$ is

$$
v(x, \ell)=\sum_{\alpha \in \mathbb{L}} r^{(\alpha)} p^{(\alpha)}(x, \ell)=r^{(\ell)} p(x, \ell)
$$

with $p^{(\alpha)}(x, \ell)=\delta_{\alpha}(\ell) p(x, \ell)$.

Proof. See Appendix D.

Proposition 5. Given an arbitrary LMO density with the pa-

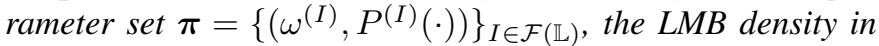
the class defined in (7) which minimizes the Kullback-Leibler divergence from $\boldsymbol{\pi}$, and preserves the first-order moment of $\boldsymbol{\pi}$ is given by

$$
\hat{\boldsymbol{\pi}}_{\mathrm{LMB}}=\left\{\left(\hat{r}^{(\alpha)}, \hat{p}^{(\alpha)}(\cdot)\right)\right\}_{\alpha \in \mathbb{L}}
$$

where

$$
\begin{aligned}
\hat{r}^{(\alpha)} & =\sum_{I \in \mathcal{F}(\mathbb{L})} 1_{I}(\alpha) \omega(I) \\
\hat{p}^{(\alpha)}(x, \ell) & =\frac{1}{\hat{r}^{(\alpha)}} \sum_{I \in \mathcal{F}(\mathbb{L})} 1_{I}(\ell) \omega(I) \delta_{\alpha}(\ell) p_{I-\{\ell\}}(x, \ell) .
\end{aligned}
$$

The density $\hat{\pi}_{\mathrm{LMB}}$ is referred to as the "best" LMB approximation of $\pi$.

Proof. See Appendix E.

\section{B. Recursive Equations of the LMB-GOM Filter}

In this subsection, we apply the derived "best" LMB approximation to the labeled multi-object filtering problem, and develop the LMB-GOM filter. The following proposition provides the update equations of the LMB-GOM filter.

Proposition 6. Suppose that the current multi-object predicted density is an $L M B$ density with the $L M B$ parameters $\pi_{+}=$ $\left\{\left(r_{+}^{\left(\alpha_{+}\right)}, p_{+}^{\left(\alpha_{+}\right)}(\cdot)\right)\right\}_{\alpha_{+} \in \mathbb{L}_{+}}$. Under a generic multi-object likelihood $g(\Upsilon \mid \mathbf{X})$, the best "LMB" approximation of the multiobject posterior is $\hat{\boldsymbol{\pi}}(\cdot \mid \Upsilon)=\left\{\left(\hat{r}^{\left(\alpha_{+}\right)}(\Upsilon), \hat{p}^{\left(\alpha_{+}\right)}(\cdot ; \Upsilon)\right)\right\}_{\alpha_{+} \in \mathbb{L}_{+}}$, where

$$
\begin{aligned}
\hat{r}^{\left(\alpha_{+}\right)}(\Upsilon) & =\sum_{I_{+} \in \mathcal{F}\left(\mathbb{L}_{+}\right)} 1_{I_{+}}\left(\alpha_{+}\right) \omega\left(I_{+} ; \Upsilon\right) \\
\hat{p}^{\left(\alpha_{+}\right)}(x, \ell ; \Upsilon) & =\frac{\delta_{\alpha_{+}}(\ell)}{r^{\left(\alpha_{+}\right)}(\Upsilon)} \sum_{I_{+} \in \mathcal{F}\left(\mathbb{L}_{+}\right)} 1_{I_{+}}(\ell) \omega\left(I_{+} ; \Upsilon\right) p_{I_{+}-\{\ell\}}(x, \ell ; \Upsilon)
\end{aligned}
$$

with

$$
\begin{aligned}
p_{\left\{\ell_{1}, \cdots, \ell_{n}\right\}}(x, \ell ; \Upsilon) & =\int P\left(\left\{(x, \ell),\left(x_{1}, \ell_{1}\right), \cdots,\right.\right. \\
\omega\left(I_{+} ; \Upsilon\right) & =\frac{\left.\left.\left(x_{n}, \ell_{n}\right)\right\} ; \Upsilon\right) d\left(x_{1}, \cdots, x_{n}\right)}{\sum_{I_{+} \in \mathcal{F}\left(\mathbb{L}_{+}\right)} \eta_{\Upsilon}\left(I_{+}\right) \omega_{+}\left(I_{+}\right)} \\
P\left(\mathbf{X}_{+} ; \Upsilon\right) & =\frac{\left[p_{+}\right]^{\mathbf{X}} g\left(\Upsilon \mid \mathbf{X}_{+}\right)}{\eta_{\Upsilon}\left(\mathcal{L}\left(\mathbf{X}_{+}\right)\right)} \\
\omega_{+}\left(I_{+}\right) & =\prod_{i \in \mathbb{L}_{+}}\left(1-r_{+}^{(i)}\right) \prod_{\ell \in I_{+}} \frac{1_{\mathbb{L}_{+}}(\ell) r_{+}^{(\ell)}}{1-r_{+}^{(\ell)}}
\end{aligned}
$$

$$
\begin{array}{r}
\eta_{\Upsilon}\left(\left\{\ell_{1}, \ell_{2}, \cdots, \ell_{n}\right\}\right)=\int g\left(\Upsilon \mid\left\{\left(x_{1}, \ell_{1}\right), \cdots,\left(x_{n}, \ell_{n}\right)\right\}\right) \\
\left(\prod_{i=1}^{n} p_{+}\left(x_{i}, \ell_{i}\right)\right) d\left(x_{1}, \cdots, x_{n}\right) .
\end{array}
$$

Proof. See Appendix F.

Proposition 6 explicitly describes how to calculate the posterior LMB parameters $\left\{\left(\hat{r}^{\left.\left(\alpha_{+}\right)\right)}(\Upsilon), \hat{p}^{\left(\alpha_{+}\right)}(\cdot ; \Upsilon)\right\}_{\ell \in \mathbb{L}_{+}}\right.$from the predicted multi-Bernoulli parameters $\left\{\left(r_{+}^{\left(\alpha_{+}\right)}, p_{+}^{\left(\alpha_{+}\right)}(\cdot)\right)\right\}_{\ell \in \mathbb{L}_{+}}$. The update stage of the LMB-GOM filter has three steps: - Write the predicted LMB density in the general LMO density form, i.e., $\boldsymbol{\pi}_{+}(\mathbf{X})=\Delta(\mathbf{X}) \omega_{+}(\mathcal{L}(\mathbf{X}))\left[p_{+}\right]^{\mathbf{X}}$;

- Compute the full multi-object posterior density $\boldsymbol{\pi}\left(\mathbf{X}_{+} \mid \Upsilon\right)$ from the general LMO density form according to Proposition 2. resulting in $\boldsymbol{\pi}\left(\mathbf{X}_{+} \mid \Upsilon\right)=\omega\left(\mathcal{L}\left(\mathbf{X}_{+}\right) ; \Upsilon\right) P\left(\mathbf{X}_{+} ; \Upsilon\right)$; 
- Approximate $\boldsymbol{\pi}\left(\mathbf{X}_{+} \mid \Upsilon\right)$ with its "best" LMB approximation $\hat{\boldsymbol{\pi}}(\cdot \mid \Upsilon)$ according to Proposition 5

Remark 4. From Propositions 5 and 6, we can deduce that in the LMB filter proposed in [18], the LMB RFS which matches the first-order moment of the $\delta-G L M B$ posterior, also minimizes the KLD from the $\delta$-GLMB posterior, among all the $L M B$ densities.

Utilizing Proposition 6 and the prediction equations of the LMB filter in [18], we can obtain the recursive equations of the LMB-GOM filter. Under the standard object motion model, the multi-object predicted density $\boldsymbol{\pi}_{+}$is an LMB density if the multi-object prior is an LMB density [18]. Moreover, based on Proposition 6, the multi-object posterior density $\boldsymbol{\pi}(\cdot \mid \Upsilon)$ can be approximated as a principled LMB density under the GOM, if the multi-object predicted density $\boldsymbol{\pi}_{+}$is an LMB density. The specified prediction and update steps of the LMB-GOM filter are given via the following:

LMB prediction: Given the current prior LMB density with the LMB parameters $\pi=\left\{\left(r^{(\alpha)}, p^{(\alpha)}(\cdot)\right)\right\}_{\alpha \in \mathbb{L}}$ and the LMB multi-object birth with the LMB parameters $\boldsymbol{\pi}_{B}=\left\{\left(r_{B}^{\left(\alpha^{\prime}\right)}, p_{B}^{\left(\alpha^{\prime}\right)}(\cdot)\right)\right\}_{\alpha^{\prime} \in \mathbb{B}}$, the multi-object prediction is another LMB density on state space $\mathbb{X}$ and finite label space $\mathbb{L}_{+}=\mathbb{B} \cup \mathbb{L}$ given by

$$
\boldsymbol{\pi}_{+}=\left\{\left(r_{+, S}^{(\alpha)}, p_{+, S}^{(\alpha)}(\cdot)\right)\right\}_{\alpha \in \mathbb{L}} \cup\left\{\left(r_{B}^{\left(\alpha^{\prime}\right)}, p_{B}^{\left(\alpha^{\prime}\right)}(\cdot)\right)\right\}_{\alpha^{\prime} \in \mathbb{B}}
$$

where

$$
\begin{aligned}
r_{+, S}^{(\alpha)} & =\eta_{S}^{(\alpha)} r^{(\alpha)} \\
p_{+, S}^{(\alpha)}\left(x_{+}, \ell_{+}\right) & =\delta_{\alpha}\left(\ell_{+}\right)\left\langle p_{S}(\cdot) f_{+}\left(x_{+} \mid \cdot\right), p^{(\alpha)}(\cdot)\right\rangle / \eta_{S}^{(\alpha)} \\
\eta_{S}^{(\alpha)} & =\left\langle p_{S}(\cdot), p^{(\alpha)}(\cdot)\right\rangle .
\end{aligned}
$$

LMB update: Given the current predicted LMB density $\boldsymbol{\pi}_{+}=\left\{\left(r_{+}^{\left(\alpha_{+}\right)}, p_{+}^{\left(\alpha_{+}\right)}(\cdot)\right)\right\}_{\alpha_{+} \in \mathbb{L}_{+}}$and the generic multi-object likelihood function $g(\Upsilon \mid \cdot)$, the approximate multi-object posterior is another LMB density $\hat{\boldsymbol{\pi}}(\cdot ; \Upsilon)=$ $\left\{\left(\hat{r}^{\left(\alpha_{+}\right)}(\Upsilon), \hat{p}^{\left(\alpha_{+}\right)}(\cdot ; \Upsilon)\right)\right\}_{\alpha_{+} \in \mathbb{L}_{+}}$computed by 53 and 54.

Remark 5. Compared with the $\delta$-GLMB-GOM filter, the LMB-GOM filter involves less computation in its prediction step because it not only reduces the integration space to single-object space, but also involves a number of integrals that increases linearly with the object number. Actually, the computational efficiency of the LMB-GOM filter can be achieved because the "best" LMB approximation completely loses correlation between object states, while the "best" $\delta$ GLMB approximation still preserves part of the correlation between object states. The $\delta-G L M B$ density has the ability to depict the statistical dependence between points [19]. However, unlike the general LMO density, the points in $a \delta$ GLMB RFS are assumed statistically independent conditional on their existences with a set of distinct labels. This assumption can lead to a scarification of some part of information on correlation between object states when approximating the full multi-object posterior as the "best" $\delta$-GLMB approximation. As for the LMB density, the points (including object states and their labels) are assumed to be statistically independent.
Hence, information on correlation between object states is completely discarded when approximating the full multi-object posterior as the "best" LMB approximation.

\section{The SMC Implementation of the LMB-GOM Filter}

Suppose that the current LMB prior is parameterised by $\boldsymbol{\pi}=\left\{\left(r^{(\alpha)}, p^{(\alpha)}(\cdot)\right)\right\}_{\alpha \in \mathbb{L}}$, where each single object density $p^{(\alpha)}(x, \ell)$ is approximated by a set of weighted particles.

At the prediction stage, for each label $\alpha \in \mathbb{L}$ of the surviving objects, the predicted existence probability $r_{+, S}^{(\alpha)}$ and the probability density $p_{+, S}^{(\alpha)}(\mathbf{x})$ are evaluated using the particles and the corresponding weights of $p^{(\alpha)}(x, \ell)$. For explicit calculation formulas, refer to the SMC implementation of multi-Bernoulli filter [12].

At the update stage, in the first place, we evaluate the parameter set $\left\{\omega^{\left(I_{+}\right)}, P^{\left(I_{+}\right)}(\mathbf{X} ; \Upsilon)\right\}_{I_{+} \subseteq \mathbb{L}_{+}}$of the full multi-object posterior $\boldsymbol{\pi}_{+}(\mathbf{X} \mid \Upsilon)$. Similar to the SMC implementation of the LMO-GOM filter presented in Subsection III-B, for each label set $I_{+} \subseteq \mathbb{L}_{+}$, the multi-object density $P^{\left(I_{+}\right)}(\mathbf{X} ; \Upsilon)$ is approximated by a set of weighted particles $\left\{\left(w_{j_{+}}^{\left(I_{+}\right)}, \mathbf{X}_{j_{+}}^{\left(I_{+}\right)}\right)\right\}_{j_{+}=1}^{N_{p}^{\left(I_{+}\right)}}$, where each particle $\mathbf{X}_{j_{+}}^{\left(I_{+}\right)}$for $j=1, \cdots, N_{p}^{\left(I_{+}\right)}$is drawn from a properly designed importance density.

Then for each label $\alpha_{+} \in \mathbb{L}_{+}$, the updated LMB parameters $\hat{r}^{\left(\alpha_{+}\right)}(\Upsilon)$ and $\hat{p}^{\left(\alpha_{+}\right)}\left(x_{+}, \ell_{+} ; \Upsilon\right)$ can be calculated from the parameter set $\left\{\omega^{\left(I_{+}\right)}, P^{\left(I_{+}\right)}(\mathbf{X} ; \Upsilon)\right\}_{I_{+} \subseteq \mathbb{L}_{+}}$utilizing the particles and the corresponding weights of each $P^{\left(I_{+}\right)}(\mathbf{X} ; \Upsilon)$. A key term when calculating the single object density $p^{(\alpha)}(x, \ell ; \Upsilon)$ is $\delta_{\alpha_{+}}(\ell) p_{I_{+}-\{\ell\}}(x, \ell)$ in 54. By utilizing (55) and (37), this term is evaluated as,

$$
\begin{aligned}
& \delta_{\alpha_{+}}(\ell) p_{I_{+}-\{\ell\}}(x, \ell) \\
= & \delta_{\alpha_{+}}(\ell) \int P^{\left(I_{+}\right)}\left(\mathbf{X}_{+} \cup \mathbb{X} \times\{\ell\} ; \Upsilon\right) \delta \mathbf{X}_{+} \\
\propto & \delta_{\alpha_{+}}(\ell) \sum_{j_{+}=1}^{N_{p}^{\left(I_{+}\right)}} \widetilde{\omega}_{j_{+}}^{\left(I_{+}\right)} \int \delta_{\mathbf{X}_{j_{+}}^{\left(I_{+}\right)}}\left(\mathbf{X}_{+} \cup \mathbb{X} \times\{\ell\}\right) \delta \mathbf{X}_{+} \\
\propto & \sum_{j_{+}=1}^{N_{p}^{(I+)}} \widetilde{\omega}_{j_{+}}^{\left(I_{+}\right)} \delta_{\mathbf{X}_{j}^{\left(I_{+}\right)} \cap \mathbb{X} \times\left\{\alpha_{+}\right\}}\left(x_{+}, \ell_{+}\right) .
\end{aligned}
$$

After the update step, the resampling and truncation processes are also applied similar to the SMC implementation of the LMO-GOM filter.

\section{Grouping based LMB-GOM Filter}

The proposed LMB-GOM filter can be seen as an extension of the LMB filter proposed in [18] that accommodates generic multi-object likelihood. To enhance the implementation efficiency of the LMB filter for the standard observation model, the parallel group update via the construction of the so called "groups" was proposed in [18]. Each group contains only closely spaced objects and their associated measurements. This method can achieve significant reductions in computation because updating independent groups in parallel is usually much faster than updating the entire multi-target state. In this subsection, we also extend the parallel group update to the LMB-GOM filter. Combining the prediction step of the LMBGOM filter with the parallel group update leads to a variant of 
the LMB-GOM filter, called the grouping based LMB-GOM (G-LMB-GOM) filter.

In this subsection, the observation set is considered as an RFS $Z$ defined on the observation space $\mathbb{Z}$. By exploiting the mathematical formulation of LMB RFSs, the LMB predicted density $\boldsymbol{\pi}_{+}=\left\{\left(r^{(\alpha)}, p^{(\alpha)}(\cdot)\right)\right\}_{\alpha \in \mathbb{L}_{+}}$admits an exact decomposition based an arbitrary partition of tracks in the label space $\mathbb{L}_{+}$, denoted by $\left\{\mathbb{L}_{+}^{1}, \cdots, \mathbb{L}_{+}^{N}\right\}$, i.e.,

$$
\boldsymbol{\pi}_{+}(\mathbf{X})=\prod_{i=1}^{N} \boldsymbol{\pi}_{+}^{i}\left(\mathbf{X} \cap \mathbb{X} \times \mathbb{L}_{+}^{i}\right)
$$

where $\boldsymbol{\pi}_{+}^{i}=\left\{\left(r^{(\alpha)}, p^{(\alpha)}(\cdot)\right)\right\}_{\alpha \in \mathbb{L}_{+}^{i}}$. The decomposition in 65 is achieved by utilizing the independence between Bernoulli components and the convolution formula given in [1, p.385].

Having the flexible decomposition of the LMB prediction, as long as there exist one partition of the tracks such that the multi-object likelihood can be decomposed as

$$
g(Z \mid \mathbf{X})=g\left(Z^{0} \mid \emptyset\right) \prod_{i=1}^{N} g\left(Z^{i} \mid \mathbf{X} \cap \mathbb{X} \times \mathbb{L}_{+}^{i}\right),
$$

where $Z^{i} \subseteq Z$ for $i=1, \cdots, N$ denotes the observation subset associated with the tracks in $\mathbb{L}_{+}^{i}$ and $Z^{0}=Z-\cup_{i=1}^{N} Z^{i}$, then the parallel group update can be achieved, i.e.,

$$
\begin{aligned}
& \boldsymbol{\pi}(\mathbf{X} \mid Z) \propto \boldsymbol{\pi}_{+}(\mathbf{X}) g(Z \mid \mathbf{X}) \\
= & \prod_{i=1}^{N} \boldsymbol{\pi}_{+}^{i}\left(\mathbf{X} \cap \mathbb{X} \times \mathbb{L}_{+}^{i}\right) \prod_{i=1}^{N} g\left(Z^{i} \mid \mathbf{X} \cap \mathbb{X} \times \mathbb{L}_{+}^{i}\right) \\
\propto & \prod_{i=1}^{N} \boldsymbol{\pi}^{(i)}\left(\mathbf{X} \cap \mathbb{X} \times \mathbb{L}_{+}^{i} \mid Z^{i}\right) .
\end{aligned}
$$

The decomposition of the multi-object likelihood in 66 essentially demands that the effects of different multi-object subsets $\mathbf{X} \times \mathbb{X} \cap \mathbb{L}_{+}^{i}$ on the observations can be separated. Specifically, each observation subset $Z^{i}$ is only correlated with the multi-object subset $\mathbf{X} \cap \mathbb{L} \times \mathbb{L}_{+}^{i}$. Nevertheless, this demand is not necessarily valid for the GOM. Hence, in the following, we firstly discuss the constrains on the observation model. Then we provide a principled method to partition tracks and observations for which the decomposition in 66 holds approximately. Finally, the parallel group update is formulated.

1) Decomposition of the Likelihood: The following assumptions on the observation model are made.

A.1: The observations $z \in Z$ are conditionally independent under the multi-object state $\mathbf{X}$;

A.2: The object with state $\mathrm{x}$ only contributes to the observations within a region $T(\mathbf{x}) \subset \mathbb{Z}$.

The first assumption is common in multi-object tracking (see, for example, [13], [26], [30]). The second assumption indicates that each observation $z \in Z$ is generated by a set of objects $\mathbf{X}_{z}=\{\mathbf{x} \in \mathbf{X}: z \in T(\mathbf{x})\}\left(\mathbf{X}_{z}\right.$ can also be an empty set). $T(\mathbf{x})$ is referred to as the valid observation region (VOR) of object $\mathbf{x}$, and $T(\mathbf{X}) \triangleq \cup_{\mathbf{x} \in \mathbf{X}} T(\mathbf{x})$ is referred to as the VOR of the state set $\mathbf{X}$. The VOR is related to the sensing characteristic of a sensor.

Proposition 7. Given an observation model characterized by multi-object likelihood $g(Z \mid \mathbf{X})$, and satisfing Assumptions A.1 and A.2, if a subset of object states, $\mathbf{X}^{\prime} \subseteq \mathbf{X}$ satisfies

$$
T\left(\mathbf{X}^{\prime}\right) \cap T\left(\mathbf{X}-\mathbf{X}^{\prime}\right)=\emptyset,
$$

then the observation subset $Z \cap T(\mathbf{X})$ is statistically independent of object states $\mathbf{X}-\mathbf{X}^{\prime}$, and the observation subset
$Z-T(\mathbf{X})$ is statistically independent of object states $\mathbf{X}$, i.e., the multi-object likelihood can be represented as

$$
g(Z \mid \mathbf{X})=g\left(Z \cap T\left(\mathbf{X}^{\prime}\right) \mid \mathbf{X}^{\prime}\right) g\left(Z-T\left(\mathbf{X}^{\prime}\right) \mid \mathbf{X}-\mathbf{X}^{\prime}\right)
$$

Herein, $\mathbf{X}^{\prime}$ is called as an isolated object cluster.

Proof. See Appendix G.

According to Proposition 7, as long as one isolated object cluster arises, the multi-object likelihood can be further decomposed as (69). Observing (68), one can easily obtain that the smaller the size of the VOR $T(\mathbf{x})$ is, the more likely it is for an isolated object cluster to arise. Generally, the sensor models can be divided into three categories in terms of different types of VORs.

- Type I: Completely confined VOR. The size of $T(\mathbf{x})$ is relatively small compared with the observation space $\mathbb{Z}$, namely, an object $\mathbf{x}$ can only affect the observations in a very limited region, and then the contribution of $\mathrm{x}$ on the observations beyond this region is zero. For example, in video tracking [4], [13], [34], a rigid body can only occupy several pixels of its surroundings. For this category of sensors, it is easy to produce isolated object clusters and then exactly decompose the likelihood according to Proposition 77. The pixeled TBD observation model employed in subsection III-E belongs to this type.

Except for Type I sensors, there also exist sensors whose VOR is the whole observation space (or a region having a comparable size with the whole observation space). Hence, all the objects contribute to almost all the observations, making the observations correlated with all the objects. These sensors can be further classified into two types as follow.

- Type II: Approximately confined VOR. Correlation between observation $z$ and object $\mathrm{x}$ decays as the "distance" between $z$ and $\mathbf{x}$ increases. The acoustic sensor network observation model [33] shown in Section III-E is a typical example. When the distance $\left\|\rho(\mathbf{x})-\xi_{m}\right\|$ between the sensor and the object is sufficiently large, the received sound amplitude at sensor $\xi_{m}$ due to the object $\mathrm{x}$ decays rapidly according to $\frac{A}{\left\|\rho(\mathbf{x})-\xi_{m}\right\|}$. Hence, the contribution of the object $\mathbf{x}$ to the observation at sensor $m$ can be negligible. Consequently, by suitably truncating the complete VOR, the decomposition of the multi-object likelihood according to 69) can be achieved with an affordble approximation error.

- Type III: Full VOR. In this case, observations are strongly correlated to all the objects, and the decomposition of likelihood is not possible. For instance, when estimating the slowly diffusing sources using a sensor network, the received observations at a certain sensor are strongly affected by all the remote sources [39].

2) Grouping and Parallel Group Update: For the standard observation model, track grouping is based on a standard gating procedure which also partitions the observation set [18], [40]. Inspired by this, this subsection provides a principled method to construct independent groups of tracks and observations for a wide variety of observation models. The following two definitions will be used in formulating our method.

Definition 3. Let $f_{\Theta}(x)$ be a density function of a random variable $\Theta$. A measurable subset of the sample space $\mathbb{O}$ of $\Theta$, 
denoted by $R$ is called the highest density region (H.D.R.) of confidence $\lambda$ if

a) $\operatorname{Pr}\{\Theta \in R\}=\int_{R} f_{\Theta}(x) d x=\lambda$;

b) for $x_{1} \in R$ and $x_{2} \notin R, f_{\Theta}\left(x_{1}\right) \geqslant f_{\Theta}\left(x_{2}\right)$.

Remark 6. The concept of H.D.R. is provided in [41], [42]. The posterior density for every point inside the H.D.R. is greater than that for every point outside of region. Thus, the region includes the more probable values of $\Theta$. Usually, the confidence $\lambda$ is set to be very close to one, e.g. $\lambda=0.99$. Thus $f_{\Theta}(x)$ is negligible for $x \notin R$ and can be approximated with 0 .

Definition 4. Consider an $L M B$ density with the $L M B$ parameters $\boldsymbol{\pi}=\left\{\left(r^{(\alpha)}, p^{(\alpha)}(\cdot)\right)\right\}_{\alpha \in \mathbb{L}}$. Denote the H.D.R. of confidence $\lambda$ for $p^{(\alpha)}(\cdot)$ by $\overline{\mathbb{X}}^{(\alpha)}$, with $\overline{\mathbb{X}}^{(\alpha)} \subset \mathbb{X} \times \mathbb{L}$. $T^{(\alpha)}=\bigcup_{\mathbf{x} \in \overline{\mathbb{X}}^{(\alpha)}} T(\mathbf{x})$ is called as the VOR of track $\alpha$. Tracks $\alpha$ and $\alpha^{\prime}$ are referred to as the coupling tracks if their VORs have intersection, i.e., $T^{(\alpha)} \cap T^{\left(\alpha^{\prime}\right)} \neq \emptyset$.

Given the LMB prediction with the LMB parameters $\boldsymbol{\pi}_{+}=$ $\left\{r^{(\alpha)}, p^{(\alpha)}(\cdot)\right\}_{\alpha \in \mathbb{L}_{+}}$, the predicted label set is partitioned as $\mathbb{L}_{+}=\biguplus_{i=1: N} \mathbb{L}_{+}^{i}$ such that no track in $\mathbb{L}_{+}^{i}$ is coupled with any track in $\mathbb{L}_{+}^{j}$ for any $i \neq j$, where $\biguplus$ denotes the disjoint union. In other words, $\forall(i, j) \in[1, N]^{2}$,

$$
i \neq j \Rightarrow\left(\cup_{\alpha \in \mathbb{L}_{+}^{i}} T^{(\alpha)}\right) \bigcap\left(\cup_{\alpha^{\prime} \in \mathbb{L}_{+}^{j}} T^{\left(\alpha^{\prime}\right)}\right)=\emptyset .
$$

Accordingly, the multi-object observation set $Z$ is partitioned as $\left\{Z^{0}, Z^{1}, \cdots, Z^{N}\right\}$ where

$$
Z^{i}=Z \bigcap\left(\cup_{\alpha \in \mathbb{L}_{+}^{i}} T^{(\alpha)}\right)
$$

denotes the observation subset related to the group of tracks with label subset $\mathbb{L}_{+}^{(i)}, i=1, \cdots, N$, and

$$
Z^{0}=Z-\bigcup_{i=1}^{N} Z^{i}
$$

denotes the observation subset having no associated tracks.

The above partitions of the predicted label set and the observation set naturally produce a set of pairs

$$
\left\{\left(\mathbb{L}_{+}^{1}, Z^{1}\right), \cdots,\left(\mathbb{L}_{+}^{N}, Z^{N}\right)\right\}
$$

with each $\left(\mathbb{L}_{+}^{i}, Z^{i}\right), i=1, \cdots, N$, referred to as a group.

Consider the multi-object state $\mathbf{X} \subseteq \bigcup_{\alpha \in \mathbb{L}_{+}} \overline{\mathbb{X}}^{(\alpha)}$ with confidence $\lambda$ sufficiently large. According to Definition 4 for each group $\left(\mathbb{L}_{+}^{i}, Z^{i}\right)$, we have

$$
\cup_{\alpha \in \mathbb{L}_{+}^{i}} T^{(\alpha)} \supseteq T\left(\mathbf{X} \cap \mathbb{X} \times \mathbb{L}_{+}^{i}\right),
$$

then by the combination of 70 , the observation subset $Z^{j}$ of any other group with $j \neq i$ has the following relationship,

$$
Z^{j} \subseteq Z-\cup_{\alpha \in \mathbb{L}_{+}^{i}} T^{(\alpha)} \subseteq Z-T\left(\mathbf{X} \cap \mathbb{X} \times \mathbb{L}_{+}^{i}\right) .
$$

Under Assumption A.1, by utilizing the independence between any $Z^{j}$ and $\mathbf{X} \cap \mathbb{X} \times \mathbb{L}_{+}^{i}(i \neq j)$, the multi-object likelihood can be decomposed as

$$
g(Z \mid \mathbf{X}) \cong g\left(Z^{0} \mid \emptyset\right) \prod_{i=1}^{N} g\left(Z^{i} \mid \mathbf{X} \cap \mathbb{X} \times \mathbb{L}_{+}^{i}\right),
$$

and consequently the posterior density is decomposed as

$$
\boldsymbol{\pi}(\mathbf{X} \mid Z) \propto \prod_{i=1}^{N} \boldsymbol{\pi}^{i}\left(\mathbf{X} \cap \mathbb{X} \times \mathbb{L}_{+}^{i} \mid Z^{i}\right)
$$

where $\pi^{i}\left(\cdot \mid Z^{i}\right)$ denotes the posterior density of the $i$ th group.

For the multi-object state $\mathbf{X} \nsubseteq \cup_{\alpha \in \mathbb{L}_{+}} \overline{\mathbb{X}}^{(\alpha)}$ with confidence $\lambda$ sufficiently large, the predicted density $\boldsymbol{\pi}_{+}(\mathbf{X})=$ $\Delta(\mathbf{X}) \omega_{+}(\mathcal{L}(\mathbf{X})) p_{+}^{\mathbf{X}}$ is negligible, and consequently the corresponding posterior density $\boldsymbol{\pi}(\mathbf{X} \mid Z)$ is negligible.

As a result, the full Bayes update can be approximated as a group of parallel updates. Specifically, the LMB prediction $\boldsymbol{\pi}_{+}^{i}$ for the $i$ th group, is updated by the likelihood $g\left(Z^{i} \mid \cdot\right)$ resulting in the posterior density $\pi^{i}\left(\cdot \mid Z^{i}\right)$ of the $i$ th group.

3) Partition Criterion: An important issue of the partition procedure is the choice of the criterion used to judge whether two tracks are coupling or not. A straightforward criterion according to the previous subsections is the predicted tracks $\alpha$ and $\alpha^{\prime}$ exhibit significant coupling if their VORs have the intersection, i.e., $T^{(\alpha)} \cap T^{\left(\alpha^{\prime}\right)} \neq \emptyset$.

In practice, the criterion can be simplified by the combination of the specific observation model. Taking the two observation models provided in Section III-E as examples, we provide principled criterions as follow.

- For the pixeled TBD model, as suggested by [23]-[25], the predicted tracks $\alpha$ and $\alpha^{\prime}$ exhibit significant coupling if their distance is small, i.e.,

$$
d\left(\alpha, \alpha^{\prime}\right) \leqslant \Lambda
$$

where $\Lambda$ is a grouping threshold and $d(\cdot, \cdot)$ is a distance function which depends on the way in which observations are acquired and the statistics of the predicted tracks. A feasible distance function is

$$
d\left(\alpha, \alpha^{\prime}\right)=\left\|\hat{z}_{+}^{(\alpha)}-\hat{z}_{+}^{\left(\alpha^{\prime}\right)}\right\|
$$

where $\hat{z}_{+}^{(\alpha)}$ is the predicted position of the track $\alpha$, and $\|\cdot\|$ denotes 2-norm distance [23]-[25]. In this case, the threshold $\Lambda$ is mainly decided by both the covariance of $p^{(\alpha)}(\cdot)$ and the VOR $T(\mathbf{x})$. Analytical details of the selection of the threshold can be found in [24]. Another suitable distance function can be the Mahalanobis distance (MHD) which depicts the impacts of both state and covariance estimate, and then the threshold is mainly decided by the $\operatorname{VOR} T(\mathbf{x})$.

- For the acoustic amplitude sensor model, a feasible criterion is the predicted tracks $\alpha$ and $\alpha^{\prime}$ exhibit coupling if

$\left\{\left(z_{m}, \xi_{m}\right):\left\|\xi_{m}-\hat{z}^{(\alpha)}\right\| \leqslant \beta\right\} \cap\left\{\left(z_{m}^{\prime}, \xi_{m}^{\prime}\right):\left\|\xi_{m}^{\prime}-\hat{z}^{\left(\alpha^{\prime}\right)}\right\| \leqslant \beta\right\}$

where $\beta$ is a given threshold for which the value $\frac{A}{\beta^{\kappa}}$ is sufficiently small.

After the criterion is established, we can obtain the partition of tracks by adopting suitable clustering algorithms [43]. Then according to (71) and (72), the associated observation subset of each group can be obtained.

Remark 7. The G-LMB-GOM filter can be extended to the case of vector observations easily, because a random vector can be equivalently transformed to a labeled RFS having a constant cardinality [44].

Remark 8. If a Type III sensor is used or all tracks are too close to be isolated, then the partition of tracks and observations is not possible. In this case, the G-LMB-GOM filter degenerates to the LMB-GOM filter automatically. 


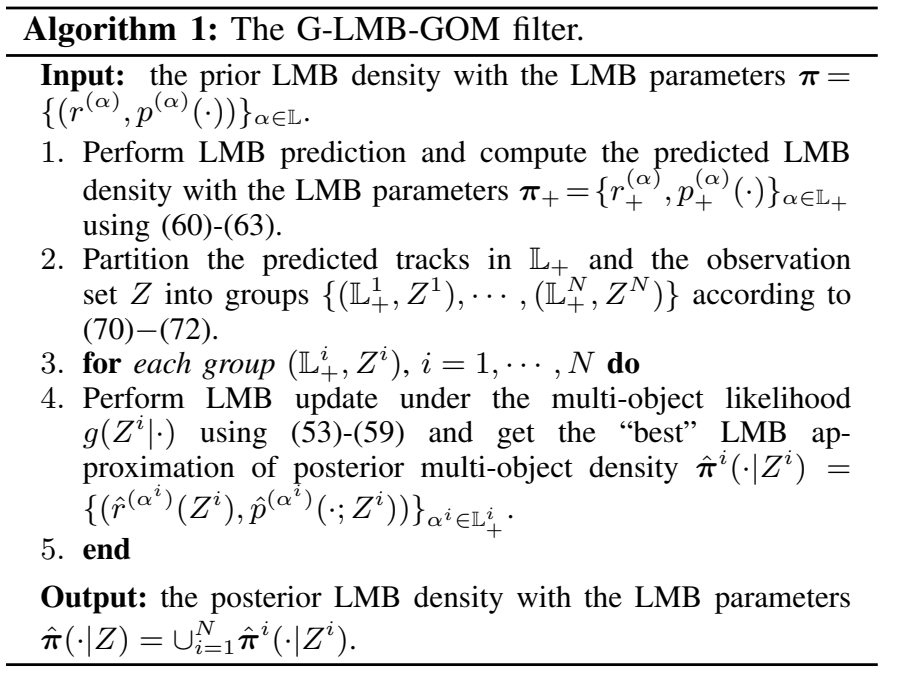

4) Summary: Algorithm 1 summarizes the steps through which the G-LMB-GOM filter can be implemented. The advantages of the G-LMB-GOM filter are two-fold:

- Firstly, it improves the computational efficiency dramatically by exploiting the parallel implementation. The detailed computational complexity is analyzed later in Section V.

- Secondly, it has the potential to improve the tracking performance especially when computing and memory resources (e.g., the number of particles that can be handled in realtime applications) are limited. On one hand, the performance compromise incurred by the grouping procedure is slight when the grouping threshold is sufficiently large. On the other hand, the densities required to be approximated (by particles) after grouping at the update stage have much lower dimensions than those in the original LMB-GOM and $\delta$ GLMB-GOM filters. Since the number of particles required to keep a certain tracking performance increases exponentially with the dimension of the state space to be sampled [24], [45], the performance improvement stemmed from the better numerical approximation of the lower dimensional densities (given a fixed number of particles) can sometimes go beyond the inaccuracy due to the grouping procedure.

\section{Computational Complexity Analyses And SCHEMATICS}

In this section, we compare the LMO-GOM, $\delta$-GLMBGOM, LMB-GOM and G-LMB-GOM filters in terms of computational complexities of their respective prediction and update equations, as shown in Table I. Fig. 1 shows how these filters operate at the conceptual level. All these algorithms can accommodate the GOM because they all embed the LMOGOM update (or the parallel group LMO-GOM update) which is an exact solution with the generic multi-object likelihood.

\section{Performance Assessment}

In this section, the performance of the proposed algorithms including the LMO-GOM, LMB-GOM, and G-LMB-GOM filters is examined and compared with the state-of-the-art in comprehensive numerical experiments. The two observation models listed in Subsection II-E, i.e., the pixeled TBD
TABLE I

COMPUTATIONAL COMPLEXITY ANALYSIS

- LMO-GOM filter: In the prediction equations, a dominant portion of computation is for calculating the quantities $\eta_{S, I}(J), J \subseteq I, I \subseteq \mathbb{L}$ in 29 which involve $\sum_{a=n}^{|\mathbb{L}|} C_{a}^{n}$ integrals each to be computed on $\mathbb{X}^{n}$ with $n$ varying from 1 to $|\mathbb{L}|$, where $C_{a}^{n}$ denotes the number of possible combinations of $n$ objects from a set of $a$ objects. In the update equation, computation is dominated by calculation of the quantities $\eta \Upsilon(I), I \subseteq \mathbb{L}_{+}$in 33 which involve computing $C_{\left|\mathbb{L}_{+}\right|}^{n}$ integrals on $\mathbb{X}^{n}$ with $n$ varying from 1 to $\left|\mathbb{L}_{+}\right|$.

- $\delta$-GLMB-GOM filter: In the prediction equations, the main part of computation is for the quantities $\eta_{S}^{(I)}(\ell), \ell \in I, I \subseteq \mathbb{L}$ which involve computing $\sum_{n=1}^{|\mathbb{L}|} n \cdot C_{|\mathbb{L}|}^{n}$ integrals on $\mathbb{X}$. In the update equation, computation is mainly for the quantities $\eta \Upsilon(I), I \subseteq \mathbb{L}_{+}$with its computational complexity being the same as that of the LMO-GOM filter.

- LMB-GOM filter: In the prediction equations, a major part of computation is for the quantities $\eta_{S}(\ell), \ell \in \mathbb{L}$ in $\sqrt{63}$, which involve computing $|\mathbb{L}|$ integrals on $\mathbb{X}$. In the update equation, the main part of computation is for the quantities $\eta_{\Upsilon}(I), I \subseteq \mathbb{L}_{+}$in 59] whose computational complexity are also the same as that of the LMO-GOM filter.

- G-LMB-GOM filter: The computational complexity of prediction equations is same as that of the LMB-GOM filter. If the label space $\mathbb{L}_{+}$is partitioned into $\left\{\mathbb{L}_{+}^{1}, \cdots, \mathbb{L}_{+}^{N}\right\}$, then the main part of computation in the update step is for calculating $N$ groups of quantities $\left\{\eta_{\Upsilon^{i}}\left(I^{i}\right), I^{i} \subseteq \mathbb{L}_{+}^{i}\right\}_{i=1}^{N}$. For a certain group $i$, it involves computing $C_{\left|\mathbb{L}_{+}^{i}\right|}^{n}$ integrals on $\mathbb{X}^{n}$ with $n$ varying from 1 to $\left|\mathbb{L}_{+}^{i}\right|$. Another computation lies in the clustering algorithm for the partition procedure. Taking the hierarchical clustering algorithm [43] as an example, the computational expense is $\mathcal{O}\left(\left|\mathbb{L}_{+}\right|^{2} \log \left(\left|\mathbb{L}_{+}\right|\right)\right)$, which is much cheaper than the computational expense for filtering.

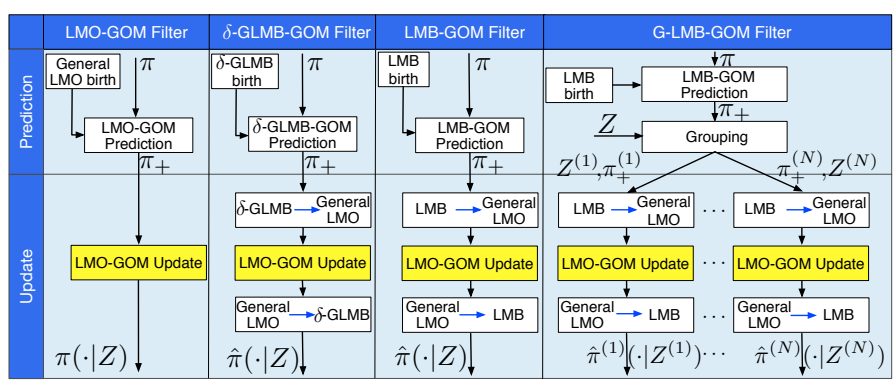

Fig. 1. Schematic presentation of how the LMO-GOM, $\delta$-GLMB-GOM, LMB-GOM and G-LMB-GOM filters operate in prediction and update steps.

model and the acoustic amplitude model are considered in our experiments. As we analysed in Subsection IV-D, these two observation models are two typical examples of Type I and Type II sensors, respectively. All the algorithms are implemented using the SMC approximation method.

The standard multi-object transition kernel provided in Section II-D is adopted. The kinematic object state variable is a vector of the plannar position and velocity $x=$

TABLE II

Particle Number of PARAMETERs For Different Algorithms

\begin{tabular}{l|l|l|l|l}
\hline \hline & $\begin{array}{l}\text { LMO-GOM } \\
\text { Filter }\end{array}$ & $\begin{array}{l}\delta \text {-GLMB- } \\
\text { GOM Filter }\end{array}$ & $\begin{array}{l}\text { LMB-GOM } \\
\text { Filter }\end{array}$ & $\begin{array}{l}\text { G-LMB- } \\
\text { GOM Filter }\end{array}$ \\
\hline \multirow{3}{*}{ Prior } & $P^{(I)}(\cdot)$ & $p^{(I, \alpha)}(\cdot)$ & $p^{(\alpha)}(\cdot)$ & $p^{(\alpha)}(\cdot)$ \\
\cline { 2 - 5 } & $2 \times 10^{6}$ & $N_{p}$ & $N_{p}$ & $N_{p}$ \\
\hline \multirow{3}{*}{ Posterior } & $P^{\left(I_{+}\right)}(\cdot ; \Upsilon)$ & $P^{\left(I_{+}\right)}(\cdot ; \Upsilon)$ & $P^{\left(I_{+}\right)}(\cdot ; \Upsilon)$ & $P^{\left(I_{+}^{i}\right)}\left(\cdot ; \Upsilon^{i}\right)$ \\
\cline { 2 - 5 } & $2 \times 10^{6}$ & $N_{p}$ & $N_{p}$ & $N_{p}$ \\
\hline
\end{tabular}


$\left[\begin{array}{llll}p_{x} & p_{y} & \dot{p}_{x} & \dot{p}_{y}\end{array}\right]^{\top}$, where “T” denotes matrix transpose. The single-object transition model is linear Gaussian with

$$
\mathbf{F}=\left[\begin{array}{cc}
\mathbf{I}_{2} & \Delta \mathbf{I}_{2} \\
\mathbf{0}_{2} & \mathbf{I}_{2}
\end{array}\right], \quad \mathbf{Q}=\sigma_{v}^{2}\left[\begin{array}{cc}
\frac{\Delta^{4}}{3} \mathbf{I}_{2} & \frac{\Delta^{3}}{2} \mathbf{I}_{2} \\
\frac{\Delta^{3}}{2} \mathbf{I}_{2} & \Delta^{2} \mathbf{I}_{2}
\end{array}\right]
$$

where $\mathbf{I}_{2}$ and $\mathbf{0}_{2}$ denote the $2 \times 2$ identity and zero matrices respectively, $\Delta=1 \mathrm{~s}$ is the sampling period, and $\sigma_{v}$ is the standard deviation of the process noise. The probability of object survival $P_{S}$ is set to be 0.98 .

The optimal sub-pattern assignment (OSPA) error [46] serves as the main performance metric with the cut-off value $c=30 \mathrm{~m}$ and the order parameter $p=1$. All performance metrics are averaged over $100 \mathrm{MC}$ runs.

\section{A. Pixeled TBD Model}

The efficacy of the proposed algorithms is first evaluated in a typical TBD scenario which presents object crossing, objects in a close proximity for a long time, and well-separated objects. Observations are collected on a $50 \times 50$ array of cells with cell lengths $\delta_{x}=\delta_{y}=1 \mathrm{~m}$. The blurring factor for the Gaussian point spread function is set to be $\delta_{b}^{2}=1$. The effective template is the $7 \times 7$ pixel square region whose center is closest to $\left(p_{x}, p_{y}\right)$. The SNR value of each object is set to be $15 \mathrm{~dB}$. Figs. 2(a) and (b) show the trajectories of five objects and an observation map at a certain time step, respectively. The duration of this scenario is $T_{s}=28 \mathrm{~s}$.

We compare our methods with the $\delta$-GLMB-GOM filter and the MB-TBD filter [13]. The SMC implementation for the MB-TBD filter adopts $5 \times 10^{4}$ particles for each Bernoulli component. The particles employed by the other algorithms are assigned according to Table II, with $N_{p}=5 \times 10^{4}$. With the G-LMB-GOM filter, we choose the partition criterion given in 75 with the distance function $(76)$, and the grouping threshold is set to be $\Lambda=10 \mathrm{~m}$.

One of the main purposes of this experiment is to verify that the LMO-GOM filter is possibly served as the theoretical performance upper bound under the standard observation model as we analysed in Remark 3 Hence, in order to guarantee a negligible numerical error with a sufficiently large but tractable number of particles (i.e., $2 \times 10^{6}$ ), the uncertainties of parameters are set to be relatively low. Specifically, all filters assume no object births and are initialized from the regions around the correct object positions. Also the five trajectories are considered with only slight maneuverability, i.e., $\sigma_{\nu}=0.01 \mathrm{~m} / \mathrm{s}^{2}$. The aim of this setting is to ensure a controlled experiment in which the objects can approach each other in a small distance for a relatively long period. The duration of this scenario is $T_{s}=60 \mathrm{~s}$.

Fig. 3 a) shows the respective outputs of the LMB-GOM and MB-TBD filters for a single MC run. It can be seen that the LMB-GOM filter performs accurately and consistently for the entire scenario in the sense that it maintains locking on all tracks and correctly estimates object positions. On the other hand, the MB-TBD filter performs considerably worse. Specifically, it loses object tracks very quickly after object crossing since object superpositions are not formulated in the MB-TBD filter.

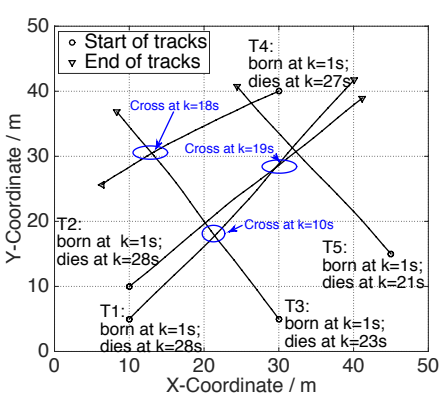

(a)

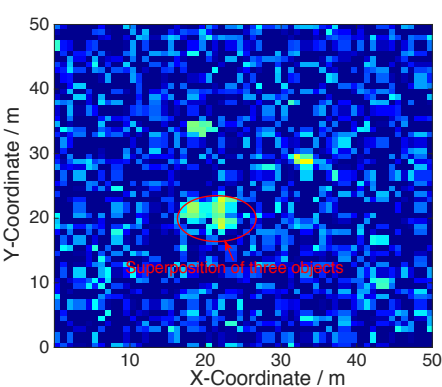

(b)
Fig. 2. (a) The trajectories of five objects in $x-y$ plane with the initial positions of objects indicated by several crosses; (b) An observation map at time $k=12 \mathrm{~s}$.

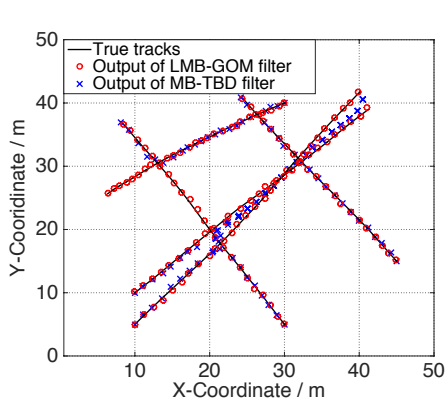

(a)

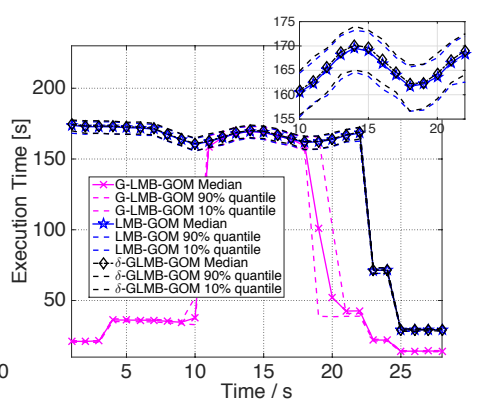

(b)
Fig. 3. (a) The respective outputs of the LMB-GOM and MB-TBD filters for a single MC run; (b) Execution time per frame for the $\delta$-GLMB-GOM, LMO-GOM and G-LMB-GOM filters.

Fig. 3(b) shows the execution times per frame for the $\delta$ GLMB-GOM, LMB-GOM and G-LMB-GOM filters. It can be seen that the execution time of the LMB-GOM filter is only slightly less than the $\delta$-GLMB-GOM filter since the scenario only considers a relative small and fixed number of objects without object birth, i.e., $|\mathbb{L}|=5$. However, due to the utilization of parallel group updates, the execution time of the G-LMB-GOM filter is dramatically less than its other competitors especially when more separated objects exist during periods $1-10 \mathrm{~s}$ and $19-28 \mathrm{~s}$.

Fig. 4 (a) shows the estimation errors over time in terms of average OSPA errors for the LMO-GOM, LMB-GOM, GLMB-GOM and $\delta$-GLMB-GOM filters. We observe comparable performance from the LMB-GOM and $\delta$-GLMB-GOM filters at all times except for the periods $7-12 \mathrm{~s}$ and $16-21 \mathrm{~s}$ during which objects the very close to each other. As the performance upper bound, the LMO-GOM filter still performs the best. Moreover, the G-LMB-GOM filter has even better performance than both the LMB-GOM and $\delta$-GLMB-GOM filter, because the grouping of objects alleviates the combinational and high-dimension problem at the update stage.

Fig. 4(b) shows average OSPA errors for the MB-TBD filter and others. The results observed are consistent with that of the single run of the MB-TBD filter. When objects are far away from each other before the time of $8 \mathrm{~s}$, the MB-TBD filter has decent accuracy, then its error begins to increase as objects get close to each other, and finally it diverges. 


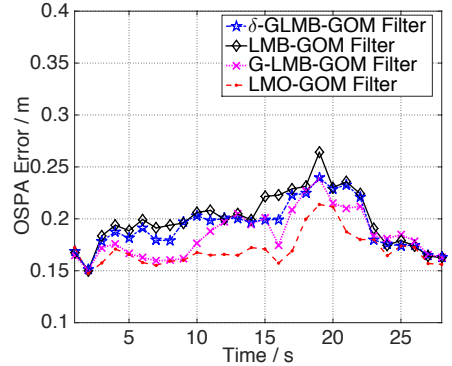

(a)

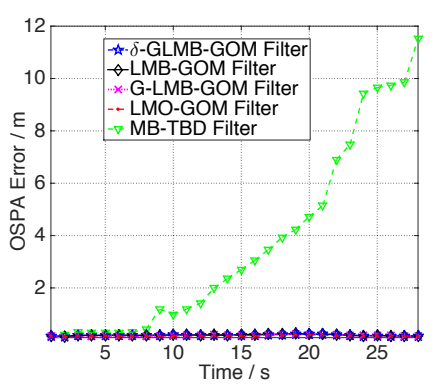

(b)
Fig. 4. (a) OSPA errors: (a) the $\delta$-GLMB-GOM, LMB-GOM, G-LMB-GOM and LMO-GOM filters; (b) all five algorithms.

\section{B. Acoustic Amplitude Model}

To further assess the capabilities of the LMB-GOM and G-LMB-GOM filter, the scenario considers the problem of tracking an unknown and time varying number of objects using acoustic amplitude sensors. A number of 961 acoustic sensors are dispersed evenly over a two-dimensional surveillance region $\left[\begin{array}{ll}0 & 300\end{array}\right] \mathrm{m} \times\left[\begin{array}{ll}0 & 300\end{array}\right] \mathrm{m}$ as shown in Fig. 5(a). At most four objects appear and travel with the standard deviation of the process noise $\sigma_{v}=0.7 \mathrm{~m} / \mathrm{s}^{2}$. The path loss exponent is set to be $\kappa=1$. The duration of this scenario is $T_{s}=60 \mathrm{~s}$.

This case is quite different from the pixeled TBD observation model in the sense that the VOR $T(\mathbf{x})$ is able to cover the whole observation space, which can be reflected from Fig. 5.b) drawing the received sound amplitude at each acoustic sensor. For the G-LMB-GOM filter, the grouping criterion (77) is utilized with the threshold $\beta=45 \mathrm{~m}$ by approximately truncating $T(\mathbf{x})$. The particles employed by each algorithm are set according to Table II with $N_{p}=10^{4}$. The birth procedure for each algorithm is as follows. At each time step, the birth process is an LMB RFS with the parameter set $\boldsymbol{\pi}_{B}=\left\{\left(r_{B}^{(i)}, p_{B}^{(i)}\right)\right\}_{i=1}^{2}$ where $r_{B}^{(i)}=0.02$ and $p_{B}^{(i)}=\mathcal{N}\left(x ; m_{B}^{(i)}, P_{B}\right)$ with $m_{B}^{(1)}=\left[\begin{array}{llll}50 & 180 & 0 & 0\end{array}\right]^{\top}$,

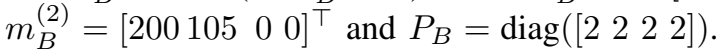

Figs. 6 and 7 show the execution times, average OSPA errors and the cardinality estimates over time for the LMB-GOM, GLMB-GOM and $\delta$-GLMB filters under the sound amplitude $A=7.9$, respectively. We observe a comparable performance from the LMB-GOM and $\delta$-GLMB-GOM filters in terms of both the cardinality estimates and the OSPA errors, while the LMB-GOM filter achieves a more evident reduction in the execution time compared to the $\delta$-GLMB-GOM filter due to the incorporation of the object birth process in this scenario. On one hand, whenever an object is born, the tracking error of the $\delta$-GLMB-GOM filter sharply increases but retracts to the

TABLE III

THE AVERAGE OSPA ERRORS (M) FOR DIFFERENT SOUND AMPLITUDES.

\begin{tabular}{c|ccc}
\hline \hline Sound Amplitude $A$ & 10 & 7.9 & 5.6 \\
\hline$\delta$-GLMB-GOM & 2.0296 & 2.4284 & 3.7133 \\
\hline LMB-GOM & 2.1012 & 2.4510 & 3.7404 \\
\hline G-LMB-GOM & 1.9852 & 2.4092 & 3.8196 \\
\hline
\end{tabular}

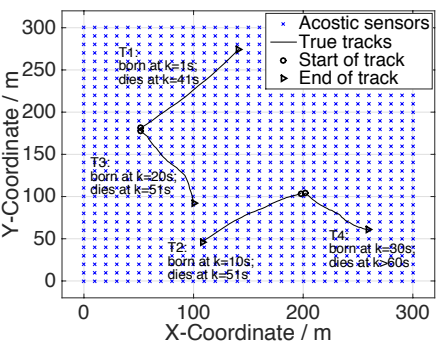

(a)

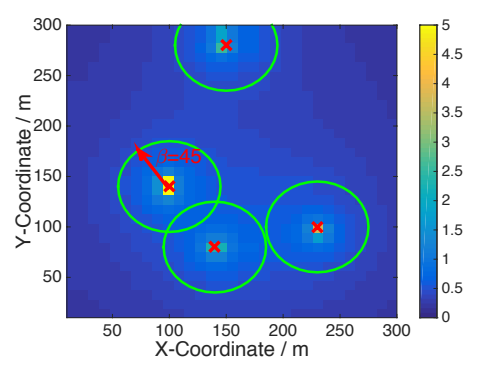

(b)
Fig. 5. (a) the trajectories of four objects in $x-y$ plane; (b) the received sound amplitude at each acoustic sensor under $A=7.9$.

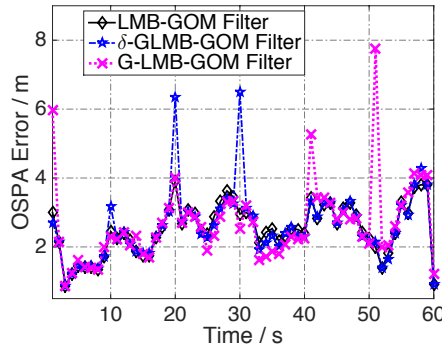

(a)

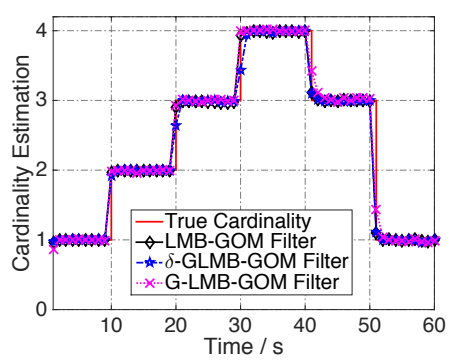

(b)
Fig. 6. Performance metrics for the $\delta$-GLMB-GOM, LMB-GOM, G-LMBGOM filters under $A=7.9$ : (a) average OSPA errors; (b) cardinality estimates and true cardinalities.

normal level quickly, while the LMB-GOM filter can handle the births of objects well. On the other hand, the tracking errors of the LMB-GOM filter are slightly higher than the $\delta$-GLMBGOM filter at the stable stage. The tracking performance of the G-LMB-GOM filter is also comparable with the other two filters. More importantly, the OSPA errors of the G-LMBGOM filter is even lower than the other two algorithms during $20 s-40 s$ when more isolated tracks have appeared. Also, the execution time for the G-LMB-GOM filter is dramatically reduced compared with the other two algorithms. However, one can also observe that when objects die (at times $40 \mathrm{~s}$ and $50 \mathrm{~s}$ ), the OSPA error of the G-LMB-GOM sharply increases but retracts to the normal level quickly, while the other two algorithms can handle the deaths of objects better. The reason is that the performance loss arising from the grouping error can be larger than the improvement in the numerical accuracy due to the parallel group update, when an object dies. The results of this experiment also verify that the G-LMB-GOM filter can also be effective for a Type II sensor.

Further, we investigate how the performances of different algorithms are affected by different values of the sound amplitude $A$. The post-transient values of the OSPA errors under $A=10,7.9,5.6$ averaged over $100 \mathrm{MC}$ runs and 60 time steps are presented in Table III.

\section{CONCLUSION}

An exact Bayesian filtering solution using labeled random finite sets, for the multi-object tracking problem under the generic observation model (GOM) and the standard transition kernel, was presented. The proposed exact solution can be 


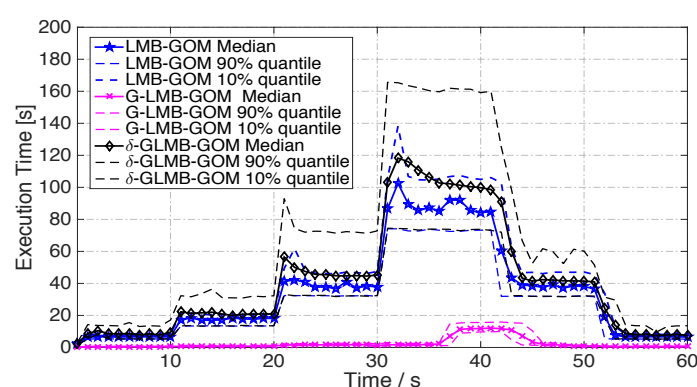

Fig. 7. Average execution times for the $\delta$-GLMB-GOM, LMB-GOM, GLMB-GOM filters under $A=7.9$.

served as the theoretical performance benchmark in multiobject tracking under the standard transition kernel. We also proposed a generalization of the LMB filter, named LMB filter for GOM (LMB-GOM filter) which is derived by approximating the full multi-object density with the closest LMB density in terms of Kullback-Leibler divergence (and it is proven to preserve the first moment as well). A variant of the LMB-GOM filter, called grouping based LMB-GOM (GLMB-GOM) filter was devised and presented through a stepby-step algorithm. The G-LMB-GOM filter can be viewed as a computationally tractable way to implement the LMB-GOM filter. The efficacy of the proposed algorithms is demonstrated using the sequential Monte Carlo implementation under two types of non-standard observation models.

Possible future works incorporate the study on the numerical implementation methods of the proposed algorithms, e.g., the unscented Kalman filter, the cubature Kalman filter.

\section{APPENDIX A}

ProOF OF PROPOSITION 1

The density of the surviving multi-object state at the next time is given by the Chapman-Kolmogorov equation

$$
\begin{aligned}
& \boldsymbol{\pi}_{S}(\mathbf{W})=\int \mathbf{f}_{S}(\mathbf{W} \mid \mathbf{X}) \boldsymbol{\pi}(\mathbf{X}) \delta \mathbf{X} \\
= & \sum_{n=0}^{\infty} \frac{1}{n !} \sum_{\left(\ell_{1}, \cdots, \ell_{n}\right) \in \mathbb{L}^{n}} \omega\left(\left\{\ell_{1}, \cdots, \ell_{n}\right\}\right) 1_{\left\{\ell_{1}, \cdots, \ell_{n}\right\}}(\mathcal{L}(\mathbf{W})) \\
\int & \prod_{i=1}^{n} \Phi\left(\mathbf{W} ; x_{i}, \ell_{i}\right) P\left(\left\{\left(x_{1}, \ell_{1}\right), \cdots,\left(x_{n}, \ell_{n}\right)\right\}\right) d\left(x_{1}, \cdots, x_{n}\right) .
\end{aligned}
$$

Substituting $P_{S,\left\{\ell, \cdots, \ell_{n}\right\}}$ of form 28 into $(79)$, we have

$$
\boldsymbol{\pi}_{S}(\mathbf{W})=\sum_{I \subseteq \mathbb{L}} 1_{I}(\mathcal{L}(\mathbf{W})) \omega(I) P_{S, I}(\mathbf{W}) .
$$

According to Definition 1 we can compute the joint probability of the label set $\left\{\ell_{+, 1}, \cdots, \ell_{+, n_{+}}\right\}$for $\boldsymbol{\pi}_{S}(\mathbf{W})$ as,

$$
\begin{aligned}
& \omega_{S}\left(\left\{\ell_{+, 1}, \cdots, \ell_{+, n_{+}}\right\}\right) \\
= & \sum_{I \subseteq \mathbb{L}} 1_{I}\left(\left\{\ell_{+, 1}, \cdots, \ell_{+, n_{+}}\right\}\right) \omega(I) \int P_{S, I}\left(\left\{\left(x_{+, 1},\right.\right.\right. \\
& \left.\left.\left.\ell_{+, 1}\right), \cdots,\left(x_{+, n_{+}}, \ell_{+, n_{+}}\right)\right\}\right) d\left(x_{+, 1}, \cdots, x_{+, n_{+}}\right) .
\end{aligned}
$$

Substitution of $\eta_{S, I}\left(\left\{\ell_{+, 1}, \cdots, \ell_{+, n_{+}}\right\}\right)$in 29 results in

$$
\begin{aligned}
& \omega_{S}\left(\left\{\ell_{+, 1}, \cdots, \ell_{+, n_{+}}\right\}\right) \\
= & \sum_{I \subseteq \mathbb{L}} 1_{I}\left(\left\{\ell_{+, 1}, \cdots, \ell_{+, n_{+}}\right\}\right) \omega(I) \eta_{S, I}\left(\left\{\ell_{+, 1}, \cdots, \ell_{+, n_{+}}\right\}\right) .
\end{aligned}
$$

Also, we can compute the joint probability density of the states $x_{+, 1}, \cdots, x_{+, n_{+}}$conditional on $\ell_{+, 1}, \cdots, \ell_{+, n_{+}}$by Definition 1 .

$P_{S}\left(\left\{\left(x_{+, 1}, \ell_{+, 1}\right), \cdots,\left(x_{+, n_{+}}, \ell_{+, n_{+}}\right)\right\}\right)=$

$\frac{\sum_{I \subseteq \mathbb{L}} 1_{I}\left(\left\{\ell_{+, 1}, \cdots, \ell_{+, n_{+}}\right\}\right) \omega(I) P_{S, I}\left(\left\{\left(x_{+, 1}, \ell_{+, 1}\right), \cdots,\left(x_{+, n_{+}}, \ell_{+, n_{+}}\right)\right\}\right)}{\omega_{S}\left(\left\{\left(\ell_{+, 1}\right), \cdots,\left(\ell_{+, n_{+}}\right)\right\}\right)}$.

Hence, $\boldsymbol{\pi}_{S}(\mathbf{W})$ can be presented as

$$
\pi_{S}(\mathbf{W})=\omega_{S}(\mathcal{L}(\mathbf{W})) P_{S}(\mathbf{W}) .
$$

For the predicted multi-object density, recall the birth density 13 , then we have

$$
\begin{aligned}
\boldsymbol{\pi}_{+}\left(\mathbf{X}_{+}\right)= & \mathbf{f}_{B}\left(\mathbf{X}_{+} \cap \mathbb{X} \times \mathbb{B}\right) \boldsymbol{\pi}_{S}\left(\mathbf{X}_{+} \cap \mathbb{X} \times \mathbb{L}\right) \\
= & \omega_{B}\left(\mathcal{L}\left(\mathbf{X}_{+}\right) \cap \mathbb{B}\right) \omega_{S}\left(\mathcal{L}\left(\mathbf{X}_{+}\right) \cap \mathbb{L}\right) \\
& \cdot P_{B}\left(\mathbf{X}_{+} \cap \mathbb{X} \times \mathbb{B}\right) P_{S}\left(\mathbf{X}_{+} \cap \mathbb{X} \times \mathbb{L}\right) .
\end{aligned}
$$

Using (24) and 25), (84) can be computed by

$$
\boldsymbol{\pi}_{+}\left(\mathbf{X}_{+}\right)=\omega_{+}\left(\mathcal{L}\left(\mathbf{X}_{+}\right)\right) P_{+}\left(\mathbf{X}_{+}\right)
$$

\section{APPENDIX B \\ PROOF OF PROPOSITION 2}

Based on the Bayes' rule, the numerator of the multi-object posterior density $\boldsymbol{\pi}(\mathbf{X} \mid \Upsilon)$ can be computed as

$$
g(\Upsilon \mid \mathbf{X}) \boldsymbol{\pi}_{+}(\mathbf{X})=g(\Upsilon \mid \mathbf{X}) \omega_{+}(\mathcal{L}(\mathbf{X})) P_{+}(\mathbf{X})
$$

Substitution of $\eta_{\Upsilon}(\cdot)$ in 33 , and $P(\mathbf{X} \mid \Upsilon)$ in 31, 86 can be further computed by

$$
\begin{aligned}
g(\Upsilon \mid \mathbf{X}) \boldsymbol{\pi}_{+}(\mathbf{X}) & =\eta \Upsilon(\mathcal{L}(\mathbf{X})) \omega_{+}(\mathcal{L}(\mathbf{X})) \frac{g(\Upsilon \mid \mathbf{X}) P_{+}(\mathbf{X})}{\eta_{\Upsilon}(\mathcal{L}(\mathbf{X}))} \\
& =\eta \Upsilon(\mathcal{L}(\mathbf{X})) \omega_{+}(\mathcal{L}(\mathbf{X})) P(\mathbf{X} ; \Upsilon)
\end{aligned}
$$

Then, the denominator of 12 can be computed by

$$
\int g(\Upsilon ; \mathbf{X}) \boldsymbol{\pi}_{+}(\mathbf{X}) \delta \mathbf{X}=\sum_{I_{+} \subseteq \mathbb{L}_{+}} \eta_{\Upsilon}\left(I_{+}\right) \omega_{+}\left(I_{+}\right) .
$$

Hence, the multi-object posterior density is

$$
\boldsymbol{\pi}(\mathbf{X} \mid \Upsilon)=\frac{\eta \Upsilon(\mathcal{L}(\mathbf{X})) \omega_{+}(\mathcal{L}(\mathbf{X})) P(\mathbf{X} ; \Upsilon)}{\sum_{I_{+} \in \mathcal{F}\left(\mathbb{L}_{+}\right)} \eta_{\Upsilon}\left(I_{+}\right) \omega_{+}\left(I_{+}\right)}=\omega(\mathcal{L}(\mathbf{X}) ; \Upsilon) P(\mathbf{X} ; \Upsilon)
$$

where $\omega\left(I_{+} ; \Upsilon\right)$ is given in 32 .

\section{APPENDIX C \\ Proof of Proposition 3}

According to the definition of the PHD [1], the labeled PHD of $\boldsymbol{\pi}(\mathbf{X})=\omega(\mathcal{L}(\mathbf{X})) P(\mathbf{X})$ can be computed as

$$
\begin{aligned}
& v(x, \ell)=\int \omega(\mathcal{L}(\{(x, \ell) \cup \mathbf{X}\})) P(\{(x, \ell)\} \cup \mathbf{X}) \delta \mathbf{X} \\
= & \sum_{n=0}^{\infty} \sum_{\left(\ell_{1}, \cdots, \ell_{n}\right) \in \mathbb{L}^{n}}\left(1-1_{\left\{\ell_{1}, \cdots, \ell_{n}\right\}}(\ell)\right) \delta_{n}\left(\left\{\ell_{1}, \cdots, \ell_{n}\right\}\right) \omega\left(\left\{\ell, \ell_{1}, \cdots, \ell_{n}\right\}\right) \\
& \cdot \frac{1}{n !} \int P\left(\left\{(x, \ell),\left(x_{1}, \ell_{1}\right), \cdots,\left(x_{n}, \ell_{n}\right)\right\}\right) d\left(x_{1}, \cdots, x_{n}\right) .
\end{aligned}
$$


Substituting $p_{\left\{\ell_{1}, \cdots, \ell_{n}\right\}}(\cdot)$ of form 499 into 89, , 89 leads to

$$
\begin{aligned}
& v(x, \ell) \\
= & \sum_{n=0}^{\infty} \frac{1}{n !} \sum_{\left(\ell_{1}, \cdots, \ell_{n}\right) \in \mathbb{L}^{n}} \delta_{n}\left(\left\{\ell_{1}, \cdots, \ell_{n}\right\}\right)\left(1-1_{\left\{\ell_{1}, \cdots, \ell_{n}\right\}}(\ell)\right) \\
\cdot \omega\left(\left\{\ell, \ell_{1}, \cdots, \ell_{n}\right\}\right) p_{\left\{\ell_{1}, \cdots, \ell_{n}\right\}}(x, \ell) & \\
= & \sum_{I \in \mathcal{F}(\mathbb{L})} 1_{I}(\ell) \omega(I) p_{I-\{\ell\}}(x, \ell) .
\end{aligned}
$$

Hence, the Proposition holds.

\section{APPENDIX D}

PROOF OF PROPOSITION 4

The LMB density can be presented as the form of (5) with

$$
\begin{aligned}
\omega(\mathcal{L}(\mathbf{X})) & =\prod_{i \in \mathbb{L}}\left(1-r^{(i)}\right) \prod_{j \in \mathcal{L}(\mathbf{X})} \frac{1_{\mathbb{L}}(j) r^{(j)}}{1-r^{(j)}} \\
P(\mathbf{X}) & =\Delta(\mathbf{X})[p]^{\mathbf{X}} .
\end{aligned}
$$

According to Proposition 3, substituting 91, and 92 in 48, we can obtain the labeled PHD of LMB density $\pi$ as,

$$
\begin{aligned}
v(x, \ell) & =\sum_{I \in \mathcal{F}(\mathbb{L})} 1_{I}(\ell) \prod_{j \in I} r^{(j)} \prod_{i \in \mathbb{L}-I}\left(1-r^{(i)}\right) p(x, \ell) \\
& =r^{(\ell)} p(x, \ell) \sum_{I^{\prime} \in \mathcal{F}(\mathbb{L}-\{\ell\})} \prod_{j \in I^{\prime}} r^{(j)} \prod_{i \in \mathbb{L}-\{\ell\}-I^{\prime}}\left(1-r^{(i)}\right) \\
& =r^{(\ell)} p(x, \ell)=\sum_{\alpha \in \mathbb{L}} r^{(\alpha)} p^{(\alpha)}(x, \ell) .
\end{aligned}
$$

Hence, the Proposition holds.

\section{APPENDIX E}

ProOf OF Proposition 5

Given an arbitrary LMO density $\boldsymbol{\pi}(\mathbf{X})=\omega(\mathcal{L}(\mathbf{X})) P(\mathbf{X})$ of the form $(5)$ on state space $\mathbb{X}$ and label space $\mathbb{L}$, we can easily obtain the LMB density $\hat{\pi}_{\mathrm{LMB}}(\mathbf{X})$ matching the labeled PHD of $\boldsymbol{\pi}(\mathbf{X})$ by comparing the labeled PHDs of the general labeled RFS and the LMB RFS shown in (48) and (50) respectively. Specifically, the parameters of $\hat{\pi}_{\mathrm{LMB}}(\mathbf{X})$ of the form (7) can be computed by

$$
\begin{aligned}
\hat{r}^{(\ell)} & =\int v(x, \ell) d x=\sum_{I \in \mathcal{F}(\mathbb{L})} 1_{I}(\ell) \omega(I) \\
\hat{p}(x, \ell) & =\frac{v(x, \ell)}{\hat{r}^{(\ell)}}=\frac{1}{\hat{r}^{(\ell)}} \sum_{I \in \mathcal{F}(\mathbb{L})} 1_{I}(\ell) \omega(I) p_{I-\{\ell\}}(x, \ell)
\end{aligned}
$$

where $v(x, \ell)$ is the labeled PHD of $\pi$.

In the following, we prove that $\hat{\pi}_{\mathrm{LMB}}$ which matches the labeled PHD of $\pi$ also minimizes the KLD from $\boldsymbol{\pi}$ over the class of LMB RFS family.

The KLD from $\pi$ and any LMB density $\overline{\boldsymbol{\pi}}_{\mathrm{LMB}}$ of the form 7. with the parameters $\bar{r}^{(\ell)}$ and $p(x, \ell)$, is given by

$$
\begin{aligned}
& D_{\mathrm{KL}}\left(\boldsymbol{\pi} ; \overline{\boldsymbol{\pi}}_{\mathrm{LMB}}\right) \\
= & \int \log \left(\frac{\omega(\mathcal{L}(\mathbf{X})) P(\mathbf{X})}{\bar{\omega}(\mathcal{L}(\mathbf{X})) \bar{P}(\mathbf{X})}\right) \omega(\mathcal{L}(\mathbf{X})) p(\mathbf{X}) \delta \mathbf{X} \\
= & \int \log \left(\frac{P(\mathbf{X})}{\bar{P}(\mathbf{X})}\right) \omega(\mathcal{L}(\mathbf{X})) P(\mathbf{X}) \delta \mathbf{X}+D_{\mathrm{KL}}(\omega ; \bar{\omega})
\end{aligned}
$$

where

$$
\begin{aligned}
\bar{\omega}(I) & =\prod_{\ell \in I} \bar{r}^{(\ell)} \prod_{\ell^{\prime} \in \mathbb{L}-I}\left(1-\bar{r}^{\left(\ell^{\prime}\right)}\right) \\
\bar{P}(\mathbf{X}) & =\Delta(\mathbf{X})[\bar{p}]^{\mathbf{X}} .
\end{aligned}
$$

Observing 96 , one can find that $D_{\mathrm{KL}}\left(\boldsymbol{\pi} ; \overline{\boldsymbol{\pi}}_{\mathrm{LMB}}\right)$ is the sum of two parts. We define the first part as

$$
C(\bar{P}) \triangleq \int \log \left(\frac{P(\mathbf{X})}{\bar{P}(\mathbf{X})}\right) \omega(\mathcal{L}(\mathbf{X})) P(\mathbf{X}) \delta \mathbf{X}
$$

and the second part as

$$
C(\bar{\omega}) \triangleq D_{\mathrm{KL}}(\omega ; \bar{\omega})
$$

First, we consider the part $C(\bar{P})$, and it can be computed by

$$
C(\bar{P})=K_{1}-\int \omega(\mathcal{L}(\mathbf{X})) P(\mathbf{X}) \sum_{\mathbf{x} \in \mathbf{X}} \log \bar{p}(\mathbf{x}) \delta \mathbf{X}
$$

where $K_{1}$ is a constant having no functional dependence on $\bar{\pi}_{\mathrm{LMB}}(\mathbf{X})$.

According to Proposition 2a in [5], i.e.,

$$
\int \sum_{y \in Y} h(y) \pi(Y) \delta Y=\int h(y) v(y) d y
$$

with $v(y)$ being the PHD of $\pi$, we have

$$
\int \omega(\mathcal{L}(\mathbf{X})) p(\mathbf{X}) \sum_{\mathbf{x} \in \mathbf{X}} \log \bar{p}(\mathbf{x}) \delta \mathbf{X}=\sum_{\ell \in \mathbb{L}} \int v(x, \ell) \log \bar{p}(x, \ell) d x
$$

According to Proposition 3, $v(x, \ell)$ has the form of (48). Substituting (48) and (102) into (100), we have

$$
C(\bar{P})=K_{1}-\sum_{\ell \in \mathbb{L}} \int \sum_{I \in \mathcal{F}(\mathbb{L})} 1_{I}(\ell) \omega(I) p_{I-\{\ell\}}(x, \ell) \log \bar{p}(x, \ell) d x .
$$

The substituting (94) and 95) into (103), we have

$$
C(\bar{P})=K_{1}+K_{2}+\sum_{\ell \in \mathbb{L}} \hat{r}^{(\ell)} D_{\mathrm{KL}}(\hat{p}(\cdot, \ell) ; \bar{p}(\cdot, \ell))
$$

where

$$
K_{2}=-\sum_{\ell \in \mathbb{L}} \hat{r}^{(\ell)} \int \hat{p}(x, \ell) \log \hat{p}(x, \ell) d x
$$

which is a constant that has no functional dependence on $\bar{p}(\cdot, \ell), \ell \in \mathbb{L}$. Hence, $C(\bar{P})$ is minimized only if $\bar{p}(\cdot, \ell)=$ $\hat{p}(\cdot, \ell)$ for each $\ell \in \mathbb{L}$.

Secondly, consider the part $C(\bar{\omega})$. According to the definition of KLD, we have

$$
\begin{aligned}
C(\bar{\omega})=K_{3}-\sum_{\ell^{\prime} \in \mathbb{L}} & \sum_{I \in \mathcal{F}(\mathbb{L})} 1_{\mathbb{L}-I}\left(\ell^{\prime}\right) \omega(I) \log \left(1-\bar{r}^{\left(\ell^{\prime}\right)}\right) \\
& -\sum_{\ell \in \mathbb{L}} \sum_{I \in \mathcal{F}(\mathbb{L})} 1_{I}(\ell) \omega(I) \log \bar{r}^{(\ell)}
\end{aligned}
$$

where $K_{3}$ is a constant independent of $\bar{\omega}(\cdot)$.

It is obvious that

$$
\sum_{I \in \mathcal{F}(\mathbb{L})} 1_{\mathbb{L}-I}\left(\ell^{\prime}\right) \omega(I)=1-\sum_{I \in \mathcal{F}(\mathbb{L})} 1_{I}\left(\ell^{\prime}\right) \omega(I)=1-\hat{r}^{\left(\ell^{\prime}\right)}
$$

with $\hat{r}^{\left(\ell^{\prime}\right)}$ shown in 94 . Thus 106 can be presented as

$$
C(\bar{\omega})=K_{3}-\sum_{\ell \in \mathbb{L}}\left(\left(1-\hat{r}^{(\ell)}\right) \log \left(1-\bar{r}^{(\ell)}\right)+\hat{r}^{(\ell)} \log \bar{r}^{(\ell)}\right) .
$$


We define two Bernoulli distributions $\hat{E}_{\ell}$ and $\bar{E}_{\ell}$ for each $\ell \in \mathbb{L}$ as

$$
\begin{aligned}
& \operatorname{Pr}\left(\hat{E}_{\ell}=1\right)=\hat{r}^{(\ell)} ; \quad \operatorname{Pr}\left(\hat{E}_{\ell}=0\right)=1-\hat{r}^{(\ell)} \\
& \operatorname{Pr}\left(\bar{E}_{\ell}=1\right)=\bar{r}^{(\ell)} ; \quad \operatorname{Pr}\left(\bar{E}_{\ell}=0\right)=1-\bar{r}^{(\ell)} .
\end{aligned}
$$

Then, Eq. 108 yields to

$$
C(\bar{\omega})=K_{3}+K_{4}+\sum_{\ell \in \mathbb{L}} D_{\mathrm{KL}}\left(\operatorname{Pr}\left(\hat{E}_{\ell}=e\right) ; \operatorname{Pr}\left(\bar{E}_{\ell}=e\right)\right)
$$

where

$$
K_{4}=-\sum_{\ell \in \mathbb{L}}\left(\left(1-\hat{r}^{(\ell)}\right) \log \left(1-\hat{r}^{(\ell)}\right)+\hat{r}^{(\ell)} \log \hat{r}^{(\ell)}\right)
$$

which is a constant having no functional dependence on any $\bar{r}^{(\ell)}, \ell \in \mathbb{L}$. Hence, $C(\bar{\omega})$ is minimized only if $\bar{r}^{(\ell)}=\hat{r}^{(\ell)}$ for each $\ell \in \mathbb{L}$.

According to $(96), D_{\mathrm{KL}}(\boldsymbol{\pi} ; \overline{\boldsymbol{\pi}})$ is minimized only if both $C(\bar{P})$ and $C(\bar{\omega})$ are minimized. Hence, $D_{\mathrm{KL}}(\boldsymbol{\pi} ; \overline{\boldsymbol{\pi}})$ is minimized by $\bar{\pi}_{\mathrm{LMB}}=\hat{\pi}_{\mathrm{LMB}}$ over the class of LMB RFS family.

\section{APPENDIX F}

\section{ProOF OF PROPOSITION 6}

Firstly, one can write the LMB prediction in the general LMO density form,

$$
\left.\boldsymbol{\pi}_{+}(\mathbf{X})=\Delta(\mathbf{X}) \omega_{+}(\mathcal{L}(\mathbf{X}))\left[p_{+}\right]^{\mathbf{X}}\right)
$$

with $\omega_{+}(\cdot)$ shown as 24 and $p_{+}(x, \ell)=p_{+}^{(\ell)}(x)$.

Then, according to Proposition 2, we can obtain the following multi-object posterior under the generic observation likelihood $g(\Upsilon \mid \mathbf{X})$,

$$
\pi(\mathbf{X} \mid \Upsilon)=\Delta(\mathbf{X}) \omega(\mathcal{L}(\mathbf{X}) ; \Upsilon) P(\mathbf{X} \mid \Upsilon)
$$

where $\omega(\cdot ; \Upsilon)$ and $P(\cdot \mid \Upsilon)$ are computed using (56) and (57), respectively.

According to Proposition 5, the LMB RFS that matches exactly the labeled first-order moment of $\boldsymbol{\pi}(\mathbf{X} \mid \Upsilon)$ as well as minimizes the Kullback-Leibler divergence from $\boldsymbol{\pi}(\mathbf{X} \mid \Upsilon)$ can be computed by

$$
\hat{\boldsymbol{\pi}}(\cdot \mid \Upsilon)=\left\{\hat{r}^{(\ell)}(\Upsilon), \hat{p}^{(\ell)}(\cdot ; \Upsilon)\right\}_{\ell \in \mathbb{L}_{+}},
$$

where $\hat{r}^{(\ell)}(\Upsilon)$ and $\hat{p}^{(\ell)}(\cdot ; \Upsilon)$ is computed by 51 and 52 .

\section{APPENDIX G}

\section{PROOF OF PROPOSITION 7}

According to Assumption A.1, for a subset of $\mathbf{X}$, denoted by $\mathbf{X}^{\prime}$, the belief mass function [1] of the observation $Z$ can be presented as

$$
\begin{aligned}
\beta(S \mid \mathbf{X}) & =\operatorname{Pr}(Z \subseteq S \mid \mathbf{X}) \\
& =\operatorname{Pr}\left(Z \cap T\left(\mathbf{X}^{\prime}\right) \subset S \mid \mathbf{X}\right) \operatorname{Pr}\left(Z-T\left(\mathbf{X}^{\prime}\right) \subset S \mid \mathbf{X}\right) .
\end{aligned}
$$

Also, according to Assumption A.2, $T\left(\mathbf{X}^{\prime}\right) \cap T\left(\mathbf{X}-\mathbf{X}^{\prime}\right)=\emptyset$ which indicates that observations $z \in T\left(\mathbf{X}^{\prime}\right)$ are generated only by object states in $\mathbf{X}^{\prime}$, and hence are independent from $\mathbf{X}-\mathbf{X}^{\prime}$, i.e.,

$$
\begin{aligned}
\operatorname{Pr}\left(Z \cap T\left(\mathbf{X}^{\prime}\right) \subseteq S \mid \mathbf{X}\right) & =\operatorname{Pr}\left(Z \cap T\left(\mathbf{X}^{\prime}\right) \subseteq S \mid \mathbf{X}^{\prime}\right) \\
& =\int_{S} 1_{T\left(\mathbf{X}^{\prime}\right)}(Z) g\left(Z \mid \mathbf{X}^{\prime}\right) \delta Z .
\end{aligned}
$$

Similarly, the observations $z \in Z-T(\mathbf{X})$ are independent from $\mathbf{X}-\mathbf{X}^{\prime}$. As a result, the belief mass function given in (116) can be calculated as follows:

$$
\begin{aligned}
\beta_{Z}(S \mid \mathbf{X})= & \int_{S} 1_{T\left(\mathbf{X}^{\prime}\right)}(Z) g\left(Z \mid \mathbf{X}^{\prime}\right) \delta Z \\
& \cdot \int_{S}\left(1-1_{T\left(\mathbf{X}^{\prime}\right)}(Z)\right) g\left(Z \mid \mathbf{X}-\mathbf{X}^{\prime}\right) \delta Z .
\end{aligned}
$$

By computing the set derivative of the above mass believe function, the multi-object likelihood can be represented as

$$
\begin{aligned}
g(Z \mid \mathbf{X})= & \sum_{Z^{\prime} \subseteq Z} 1_{T\left(\mathbf{X}^{\prime}\right)}\left(Z^{\prime}\right) g\left(Z^{\prime} \mid \mathbf{X}^{\prime}\right)\left(1-1_{T\left(\mathbf{X}^{\prime}\right)}\left(Z-Z^{\prime}\right)\right) \\
& \cdot g\left(Z-Z^{\prime} \mid \mathbf{X}-\mathbf{X}^{\prime}\right) \\
= & g\left(Z \cap T\left(\mathbf{X}^{\prime}\right) \mid \mathbf{X}^{\prime}\right) g\left(Z-T\left(\mathbf{X}^{\prime}\right) \mid \mathbf{X}-\mathbf{X}^{\prime}\right)
\end{aligned}
$$

\section{REFERENCES}

[1] R. Mahler, Statistical Multisource-Multitarget Information Fusion. Norwell, MA, USA: Artech House, 2007.

[2] V. Marmarelis and T. Berger, "General methodology for nonlinear modeling of neural systems with Poisson point-process inputs," Mathematical biosciences, vol. 196, no. 1, pp. 1-13, Jul. 2005.

[3] D. L. Snyder, L. J. Thomas, and M. M. Ter-Pogossian, "A mathematical model for Positron-emission tomography systems having time-of-flight measurement," IEEE Trans. Nuclear Science, vol. 28, no. 3, pp. 35753583, Jun. 1981.

[4] R. Hoseinnezhad, B.-N.Vo, and B.-T. Vo, "Visual tracking in background subtracted image sequences via multi-Bernoulli filtering," IEEE Trans. on Signal Process., vol. 61, no. 2, pp. 392-397, Jan. 2013.

[5] R. Mahler, "Multitarget Bayes filtering via first-order multitarget moments," IEEE Trans. on Aerosp. Electron. Syst, vol. 39, no. 4, pp. 1152 1178, Oct. 2003.

[6] _ Advances in Statistical Multisource-Multitarget Information Fusion. Norwell, MA, USA: Artech House, 2014.

[7] B. L. Wang, W. Yi, R. Hoseinnezhad, S. Q. Li, L. J. Kong, and X. B. Yang, "Distributed fusion with multi-Bernoulli filter based on generalized Covariance Intersection," IEEE Trans. on Signal Process., vol. 65 , no. 1 , pp. 242-255, Jan. 2017.

[8] J. Mullane, B. N. Vo, M. Adams, and B. T. Vo, "A random-finite-set approach to Bayesian SLAM," IEEE Trans. on Robotics, vol. 27, no. 2, pp. 268-282, Apr. 2011.

[9] B. T. Vo and W. K. Ma, "The Gaussian mixture probability hypothesis density filter," IEEE Trans. on Signal Process., vol. 54, no. 11, pp. 4091-4104, Nov. 2006.

[10] R. Mahler, "PHD filters of higher order in target number," IEEE Trans. on Aerosp. Electron. Syst., vol. 43, no. 4, pp. 1523-1543, Oct. 2007.

[11] B. T. Vo, B. N. Vo, and A. Cantoni, "Analytic implementations of the cardinalized probability hypothesis density filter," IEEE Trans. on Signal Process., vol. 55, no. 7, pp. 3553-3567, Jun. 2007.

[12] — "The cardinality balanced multi-target multi-Bernoulli filter and its implementations," IEEE Trans. on Signal Process., vol. 57, no. 2, pp. 409-423, Feb. 2009.

[13] B. T. Vo, B. N. Vo, N. T. Pham, and D. Suter, "Joint detection and estimation of multiple objects from image observations," IEEE Trans. on Signal Process., vol. 58, no. 10, pp. 5129-5141, Oct. 2010.

[14] B. T. Vo and B. N. Vo, "Labeled random finite sets and multi-object conjugate priors," IEEE Trans. on Signal Process., vol. 61, no. 13, pp. 3460-3475, Jul. 2013.

[15] B. N. Vo, B. T. Vo, and D. Phung, "Labeled random finite sets and the Bayes multi-target tracking filter," IEEE Trans. on Signal Process., vol. 62, no. 24, pp. 6554-6567, Dec. 2014.

[16] C. Fantacci, B. T. Vo, F. Papi, and B. N. Vo, "The marginalized $\delta$-GLMB filter," arXiv preprint arXiv:1501.00926, 2015

[17] M. Beard, B. T. Vo, and B. N. Vo, "Bayesian multi-target tracking with merged measurements using labelled random finite sets," IEEE Trans. Signal Process., vol. 63, no. 6, pp. 1433-1447, Aug. 2015.

[18] S. Reuter, B. T. Vo, B. N. Vo, and K. Dietmayer, "The labeled multiBernoulli filter," IEEE Trans. on Signal Process., vol. 62, no. 12, pp. 3246-3260, Jun. 2014. 
[19] F. Papi, B. N. Vo, B. T. Vo, C. Fantacci, and M. Beard, "Generalized labeled multi-Bernoulli approximation of multi-object densities," IEEE Trans. on Signal Process., vol. 63, no. 20, pp. 5487-5497, 2015.

[20] F. Papi and D. Y. Kim, "A particle multi-target tracker for superpositional measurements using labeled random finite sets," IEEE Trans. on Signal Process., vol. 63, no. 16, pp. 4348-4358, Jun. 2015.

[21] W. Yi, M. Jiang, and R. Hoseinnezhad, "The multiple model Vo-Vo filter," IEEE Trans. Aerosp. Electron. Syst., vol. 53, no. 2, pp. 10451054, Apr. 2017.

[22] S. Q. Li, W. Yi, R. Hoseinnezhad, G. Battistelli, B. L. Wang, and L. J. Kong, "Robust distributed fusion with labeled random finite sets," IEEE Trans. on Signal Process., accepted, DOI: 10.1109/TSP.2017.2760286, Sep. 2017.

[23] A. F. García-Fernández, J. Grajal, and M. R. Morelande, "Two-layer particle filter for multiple target detection and tracking," IEEE Trans. on Aerosp. Electron. Syst., vol. 49, no. 3, pp. 1569-1588, Jul. 2013.

[24] M. R. Morelande, C. M. Kreucher, and K. Kastella, "A Bayesian approach to multiple target detection and tracking," IEEE Trans. on Signal Process., vol. 55, no. 5, pp. 1589-1604, May 2007.

[25] W. Yi, M. R. Moreland, L. Kong, and J. Yang, "A computationally efficient particle filter for multitarget tracking using an independence approximation," IEEE Trans. on Signal Process., vol. 61, no. 4, pp. 843-856, Feb. 2013.

[26] W. Yi, M. Morelande, L. Kong, and J. Yang, "An efficient multi-frame track-before-detect algorithm for multi-target tracking," IEEE J. Sel. Top. Signal Process., vol. 7, no. 3, pp. 421-434, Jun. 2013.

[27] H. C. Jiang, W. Yi, T. Kirubarajan, L. J. Kong, and X. B. Yang, "Multiframe radar detection of fluctuating targets using phase information," IEEE Trans. Aerosp. Electron. Syst., vol. 53, no. 2, pp. 736-749, Apri. 2017.

[28] — , "Track-before-detect strategies for radar detection in G0distributed clutter," IEEE Trans. Aerosp. Electron. Syst., vol. PP, no. 99 , May. 2017.

[29] R. Mahler, "CPHD filters for superpositional sensors," in Proc. SPIE Optical Engineering and Applications, 2009, pp. 74 450E-74 450E.

[30] S. Nannuru, M. Coates, and R. Mahler, "Computationally-tractable approximate PHD and CPHD filters for superpositional sensors," IEEE J. Sel. Top. Signal Process., vol. 7, no. 3, pp. 410-420, Jun. 2013.

[31] S. Nannuru and M. Coates, "Hybrid multi-Bernoulli and CPHD filters for superpositional sensors," IEEE Trans. on Aerosp. Electron. Syst., vol. 51, no. 4, pp. 2847-2863, Oct. 2015.

[32] C. Lundquist, K. Granstrm, and U. Orguner, "An extended target CPHD filter and a Gamma Gaussian inverse Wishart implementation," IEEE J. Sel. Top. Signal Process., vol. 7, no. 3, pp. 472-483, Jun. 2013.

[33] O. Hlinka, O. Sluciak, F. Hlawatsch, P. M. Djuric, and M. Rupp, "Likelihood consensus and its application to distributed particle filtering," IEEE Trans. on Signal Process., vol. 60, no. 8, pp. 4334-4349, Aug. 2012.

[34] R. Hoseinnezhad, B.-N. Vo, B.-T. Vo, and D. Suter, "Visual tracking of numerous targets via multi-Bernoulli filtering of image data," Pattern Recognition, vol. 45, no. 10, pp. 3625-3635, Oct. 2012.

[35] S. Q. Li, W. Yi, B. L. Wang, and L. J. Kong, "Labeled multi-object tracking algorithms for generic observation model," in Proc. IEEE Int. Fusion Conf., Jul. 2016, pp. 1125-1131.

[36] B. Ristic, S. Arulampalam, and N. J. Gordon, Beyond the Kalman Filter: Particle Filters for Tracking Applications. Artech House, 2004.

[37] N. J. Gordon, D. J. Salmond, and A. F. M. Smith, "Novel approach to nonlinear/non-Gaussian Bayesian state estimation," in Proc. Inst. Elect. Eng. $F$, vol. 140 , no. 2, 1993, pp. 107-113.

[38] D. Crisan and A. Doucet, "Convergence of sequential Monte Carlo methods," University of Cambridge, CUED/F-INFENG/TR.381, Tech. Rep., 2000.

[39] G. Battistelli, L. Chisci, N. Forti, G. Pelosi, and S. Selleri, "Point source estimation via finite element multiple-model Kalman filtering," in 2015 IEEE 54th Annual Conference on Decision and Control (CDC), Dec., pp. 4984-4989.

[40] J. Dezert and Y. Bar-Shalom, "Joint probabilistic data association for autonomous navigation," IEEE Trans. on Aerosp. Electron. Syst., vol. 29 , no. 4, pp. 1275-1286, Oct. 1993

[41] G. E. Box and G. C. Tiao, Bayesian Inference in Statistical Analysis. Addison Wesley, 1973.

[42] R. J. Hyndman, "Computing and graphing highest density regions," The American Statistician, vol. 50, no. 2, pp. 120-126, 1996.

[43] L. Rokach and O. Maimon, "Clustering methods," in Data mining and knowledge discovery handbook. Springer US, 2005, pp. 321-352.

[44] D. Svensson, M. Guerriero, and P. Willett, "Set JPDA filter for multitarget tracking," IEEE Trans. on Signal Process., vol. 59, no. 10, pp. 4677-4691, Oct. 2011.
[45] F. Daum and J. Huang, "Curse of dimensionality and particle filters," in Proc. IEEE Aerosp. Conf., vol. 4, Mar. 2003, pp. 1979-1993.

[46] D. Schumacher, B. T. Vo, and B. N. Vo, "A consistent metric for performance evaluation of multi-object filters," IEEE Trans. on Signal Process., vol. 56, no. 8, pp. 3447-3457, Aug. 2008.

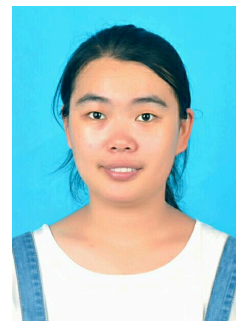

Suqi $\mathbf{L i}$ is born in 1990. She received the B.E. degree in electronic engineering from the University of Electronic Science and Technology of China, Chengdu, in 2011. Since September 2011, she has been pursuing the Ph.D. degree at the School of Electronic Engineering, University of Electronic Technology and Science of China. Currently, she is a visiting student with the Dipartimento di Ingegneria dell' Informazione (DINFO), Università degli Studi di Firenze, Italy. Her research interests include the random finite set, multi-target tracking, nonlinear filtering, sensor networks and data fusion.

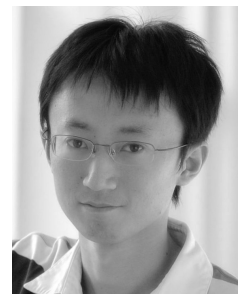

Wei Yi received the B.E. degree in electronic engineering from the University of Electronic Science and Technology of China, Chengdu, in 2006.

Since 2007, he has been pursuing the Ph.D. degree at the School of Electronic Engineering of the University of Electronic Technology and Science of China.

From March 2010 to February 2012, he was a visiting student in the Melbourne Systems Laboratory, University of Melbourne, Australia. His research interests include particle filtering and target tracking (particular emphasis on multiple target tracking and track-beforedetect techniques).

Mr. Yi received the "Best Student Paper Award" at the 2012 IEEE Radar Conference, Atlanta, United States and the "Best Student Paper Award" at the 15th International Conference on Information Fusion, Singapore, 2012.

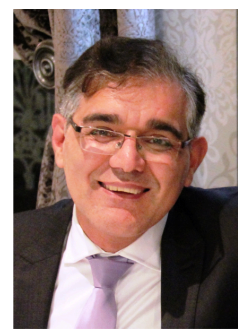

Reza Hoseinnezhad received his B.Sc., M.Sc. and Ph.D. degrees in Electronic, Control and Electrical Engineering all from the University of Tehran, Iran, in 1994, 1996 and 2002, respectively. Since 2002, he has held various academic positions at the University of Tehran, Swinburne University of Technology, the University of Melbourne and RMIT University. He is currently an Associate Professor with the School of Aerospace, Mechanical and Manufacturing Engineering, RMIT University, Victoria, Australia. His research is currently focused on development of robust estimation and visual tracking methods in a point process framework.

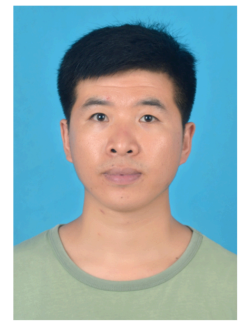

Bailu Wang received his B.S. degree from the University of Electronic Science and Technology of China (UESTC) in 2011. He is now working toward his Ph.D. degree on signal and information processing at UESTC.

From August 2016, he has been a visiting student with the Dipartimento di Ingegneria dell' Informazione (DINFO), Università degli Studi di Firenze, Italy. His current research interests include radar and statistical signal processing, and multi-sensor multitarget fusion. 


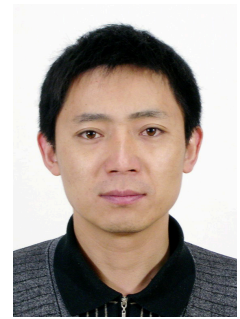

Lingjiang Kong was born in 1974. He received the B.S., M.S., and Ph.D. degrees from the University of Electronic Science and Technology of China (UESTC) in 1997, 2000 and 2003, respectively.

From September 2009 to March 2010, he was a visiting researcher with the University of Florida.

$\mathrm{He}$ is currently a professor with the School of Electronic Engineering, University of Electronic Science and Technology of China (UESTC). His research interests include multiple-input multiplestatistical signal processing. 